\title{
DIGITALCOMMONS
}

$@$ WAYNESTATE-

Wayne State University

$11-1-2007$

\section{Relative Pareto Minimizers to Multiobjective Problems: Existence and Optimality Conditions}

Truong Q. Bao

Wayne State University

Boris S. Mordukhovich

Wayne State University, boris@math.wayne.edu

\section{Recommended Citation}

Bao, Truong Q. and Mordukhovich, Boris S., "Relative Pareto Minimizers to Multiobjective Problems: Existence and Optimality Conditions" (2007). Mathematics Research Reports. Paper 55.

http://digitalcommons.wayne.edu/math_reports/55 


\title{
RELATIVE PARETO MINIMIZERS TO MULTIOBJECTIVE PROBLEMS: EXISTENCE AND OPTIMALITY CONDITIONS
}

\author{
TRUONG Q. BAO and BORIS S. MORDUKHOVICH
}

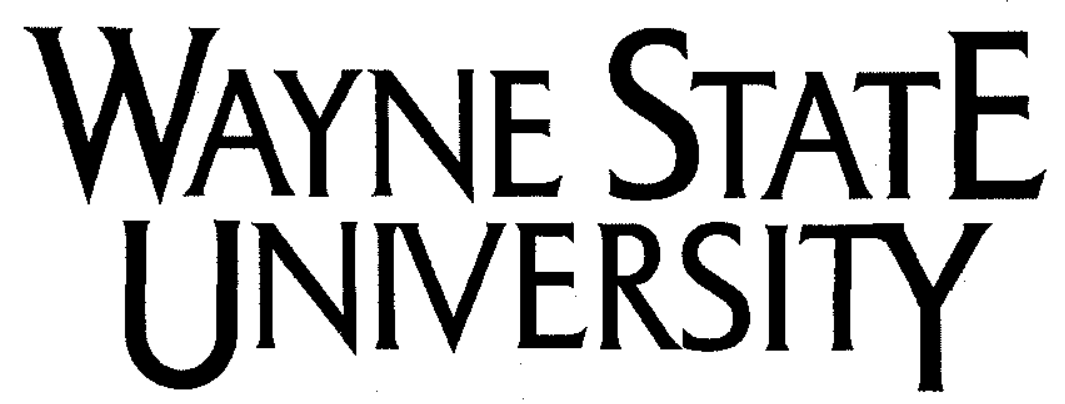

Detroit, MI 48202

Department of Mathematics

Research Report

2007 Series

\#11

This research was partly supported by the USA National Science Foundation and the Australian Research Council 


\title{
RELATIVE PARETO MINIMIZERS TO MULTIOBJECTIVE PROBLEMS: EXISTENCE AND OPTIMALITY CONDITIONS ${ }^{1}$
}

\author{
Truong Q. Bao and Boris S. Mordukhovich \\ Department of Mathematics, Wayne State University, Detroit, Michigan 48202, U.S.A. \\ tqbao@math.wayne.edu, boris@math.wayne.edu
}

\begin{abstract}
In this paper we introduce and study enhanced notions of relative Pareto minimizers to constrained multiobjective problems that are defined via several kinds of relative interiors of ordering cones and occupy intermediate positions between the classical notions of Pareto and weak Pareto efficiency/minimality. Using advanced tools of variational analysis and generalized differentiation, we establish the existence of relative Pareto minimizers to general multiobjective problems under a refined version of the subdifferential Palais-Smale condition for set-valued mappings with values in partially ordered spaces and then derive necessary optimality conditions for these minimizers (as well as for conventional efficient and weak efficient counterparts) that are new in both finite-dimensional and infinite-dimensional settings. Our proofs are based on variational and extremal principles of variational analysis; in particular, on new versions of the Ekeland variational principle and the subdifferential variational principle for set-valued and single-valued mappings in infinite-dimensional spaces.
\end{abstract}

Keywords: Multiobjective optimization, variational analysis, relative Pareto minimizers, existence theorems, necessary optimality conditions, variational and extremal principles, generalized differentiation

Mathematics Subject Classification (2000): 90C29, 49J52, 49J53

\section{Introduction}

This paper is devoted to the study of multiobjective/vector optimization problems. We consider general classes of such problems with set-valued objectives (cost mappings) and geometric constraints in infinite-dimensional spaces, while most of the results obtained seem to be new even for conventional vector optimization problems in finite dimensions.

There are various notions of solutions to multiobjective optimization problems; see, e.g., the books $[12,13,15,16,18]$ and the references therein. The classical ones relate to Pareto and weak Pareto efficient points of sets. Recall that, given a subset $\Xi$ of a normed space $Z$ partially ordered by a closed and convex cone $\Theta$ via

$$
z_{1} \leq z_{2} \Longleftrightarrow z_{2}-z_{1} \in \Theta
$$

the point $\bar{z} \in \Xi$ is a Pareto minimal/efficient point for this set if

$$
(\bar{z}-\Theta) \cap \Xi=\{\ddot{z}\} .
$$

Assuming that $\operatorname{int} \Theta \neq \emptyset$, a weak Pareto efficient/weak minimal point of $\Xi$ is defined by

$$
(\bar{z}-\operatorname{int} \Theta) \cap \Xi=\emptyset, \quad \operatorname{int} \Theta \neq \emptyset \text {. }
$$

A visible disadvantage of weak minimal points (1.3) is the nonempty interior requirement on the ordering cone $\Theta$, which seems to be a serious restriction from both viewpoints of optimization theory and applications. In particular, various vector optimization problems can be formalized

\footnotetext{
${ }^{1}$ This research was partly supported by the USA National Science Foundation under grants DMS-0304989 and DMS-0603846 and by the Australian Research Council under grant DP-9551168.
} 
by using convex ordering cones having empty interiors in both finite-dimensional and infinitedimensional settings; see, e.g., $[10,13,18]$ with more discussions, examples, and references. In such cases, the usage of appropriate relative interior points of the corresponding ordering cones seems to be reasonable provided, of course, that such points exist.

Recall that the standard relative interior of $\Theta \subset Z$, denoted ri $\Theta$, is the interior of $\Theta$ relative to the closed affine hull of $\Theta$. It is well known that ri $\Theta \neq \emptyset$ for every nonempty convex set $\Theta$ in finite dimensions. However, it is not the case in many infinite-dimensional settings. In particular, it is well known that the natural ordering cones in the Lebesgue spaces $l^{p}$ and $L^{p}$ for $1 \leq p<\infty$ and in a number of other classical infinite-dimensional spaces have empty relative interiors.

To improve this situation, some extensions of the relative interior notion have been introduced. A major extension given by Borwein and Lewis [6] under the name of quasi relative interior of $\Theta \subset Z$, with the notation qri $\Theta$, is defined as follows: qri $\Theta$ is the collection of those $z \in \Theta$ for which the closed conic hull $\overline{c o n e}(\Theta-z)$ of the set $\Theta-\bar{z}$ is a linear subspace of $Z$. It is proved in [6, Theorem 2.19] that qri $\Theta \neq \emptyset$ for any closed and convex set $\Theta \neq \emptyset$ in a separable Banach space. Further properties and various applications of quasi relative interiors of convex sets in Banach spaces can be found in $[5,6,7,8]$ and the references therein.

Another useful extension of the classical relative interior notion for convex sets in infinite dimensions has been known under the name "intrinsic core" [14] (which may be confusing; see [5]) and also under the name "pseudo relative interior" [5], which seems to be confusing as well, since "pseudo" means "false". We prefer the name intrinsic relative interior of $\Theta \subset Z$, denoted iri $\Theta$, that is defined as follows: iri $\Theta$ is the collection of those $z \in \Theta$ for which the conic hull cone $(\Theta-z)$ is a linear subspace of $Z$. One obviously has the inclusions

$$
\operatorname{ri} \Theta \subset \operatorname{iri} \Theta \subset \text { qri } \Theta \text {, }
$$

which both hold as equalities if $Z$ is finite-dimensional. Various properties of iri $\Theta$, including verifiable conditions under which iri $\Theta \neq \emptyset$ in infinite dimensions, can be found in $[5,14]$. Note that iri $\Theta=$ ri $\Theta$ in any Banach space if the affine hull of $\Theta$ is closed.

Using the afore-mentioned notions of relative interiors, we introduce now the corresponding notions of relative minimum points of sets that occupy intermediate positions between Pareto and weak Pareto minimal/efficient points. Given a subset $\Xi \subset Z$ partially ordered by the closed and convex cone $\{0\} \neq \Theta \subset Z$, we say that $\bar{z} \in \Xi$ is a (primary) relative minimal point of $\Xi$ if

$$
(\vec{z}-\operatorname{ri} \Theta) \cap \Xi=\emptyset, \quad \operatorname{ri} \Theta \neq \emptyset,
$$

that $\bar{z} \in \Xi$ is an intrinsic relative minimal point of $\Xi$ if

$$
(\bar{z}-\operatorname{iri} \Theta) \cap \Xi=\emptyset, \quad \operatorname{iri} \Theta \neq \emptyset,
$$

and that $\bar{z} \in \Xi$ is a quasi relative minimal point of $\Xi$ if

$$
(\bar{z}-\operatorname{qri} \Theta) \cap \Xi=\emptyset, \quad \text { qri } \Theta \neq \emptyset .
$$

Since both inclusions in (1.4) hold as equalities if ri $\Theta \neq \emptyset$ by [5, Theorem 2.12], all the three notions of minimal points in (1.5)-(1.7) agree if the set $\Xi$ admits a relative minimal point (1.5). Furthermore, these notions imply the weak Pareto efficiency (1.3) provided that int $\Theta \neq \emptyset$. In general, any quasi relative minimal point of $\Xi$ is an intrinsic relative minimal point of this set (but not vice versa), and the existence of the latter does not imply the existence of primary relative 
minimal points of $\Xi$ and the existence of weak efficient points of this set; the corresponding counterexamples can be easily deduced from [5].

The major goal of this paper is to study optimal solutions to constrained multiobjective optimization problems of the type:

$$
\text { minimize } F(x) \text { subject to } x \in \Omega \text {, }
$$

where the appropriate concepts of "minimality" are generated by the geometric notions of (primary, intrinsic, quasi) relative minimal points of sets defined in (1.5)-(1.7); see below for the exact definitions and more details. We consider the general setting of (1.8), where the objective $F: X \rightrightarrows Z$ is a set-valued mapping between Banach spaces with a partial order (1.1) on $Z$, and where $\Omega \subset X$ is an arbitrary closed set. However, most of the results obtained seem to be new even for classical cases of standard vector optinization problems defined by smooth single-valued mappings/functions in finite-dimensional and infinite-dimensional spaces.

We pay the main attention to establishing the existence of relative Pareto minimizers and to deriving necessary optimality conditions for them. These topics have been considered in our previous papers $[3,4]$ addressed to the conventional notions of Pareto and weak Pareto efficiency for problem (1.8). The principal results obtained in this paper allow us to extend the corresponding results of $[3,4]$ to the new notions of relative Pareto minimizers unifying also the previous results and their proofs. Moreover, the existence theorem for relative Pareto minimizers established below provides a new existence result for the case of weak minimizers under a refined version of the subdifferential Palais-Smale condition. Likewise, necessary optimality conditions derived in this paper give in addition new information for weak efficient and Pareto efficient solutions for multiobjective problems with no pointedness assumption on ordering cones. Note that in the case of Pareto minimizers (efficient solutions) we do not impose any interiority/relative interiority requirements on the ordering cone in question and alternate the "sequential normal compactness property" required for it in $[3,4]$ in infinite dimensions.

Our approach to both existence issues and necessary optimality conditions for relative Pareto minimizers is based on advanced tools of variational analysis and generalized differentiation. Besides using known results in this direction, which largely revolve around the extremal principle of variational analysis and can be found in the recent books by Mordukhovich [17, 18], we establish here new versions of the Ekeland variational principle and the subdifferential variational principle for set-valued mappings. These extensions are certainly of independent interest, while they are employed in the paper to deriving the main results on Pareto and relative Pareto minimizers.

The rest of the paper is organized as follows. In Section 2 we briefly review, for the reader's convenience, some basic tools of generalized differentiation broadly used in the paper. Besides the fundamentals from [17], they include the subdifferential constructions for set-valued mappings with values in partially ordered spaces recently introduced in [3]. Here we also present the underlying extremal principle for systems of sets, which plays a crucial role in our study.

Section 3 is devoted to extended variational principles for set-valued mappings, which are important for their own sake being crucial for establishing the subsequent results of the paper. Namely, we derive new versions of the Ekeland variational principle and the subdifferential variational principle for mappings with values in partially ordered Banach spaces. The main difference between the new versions established in this section and the most recent ones given in [3] is the usage of the so-called limiting monotonicity condition imposed on the objective mappings, which has not been previously recognized and used in variational principles for either set-valued or single-valued case. We compare the new limiting monotonicity condition with the well-known domination property previously used in [3]. 
Section 4 concerns the existence of relative Pareto minimizers to multiobjective problems. The main result here establishes the existence of intrinsic relative minimizers induced by (1.6) under a refined version of the so-called subdifferential Palais-Smale condition, which is new for both single-valued and set-valued objectives. This major result implies the existence of relative Pareto minimizers induced by (1.5) and provides also an essential improvement of the existence theorem for weak minimizers established in [3]. Our proof of the main existence theorem is based on applying the afore-mentioned extremal and variational principles together with advanced results of generalized differential calculus. Furthermore, new calculus rules for vector subgradients allow us to derive efficient specifications of the main existence theorem for multiobjective problems with explicit constraints of various types; in Section 4 we present one result in this direction for the case of general geometric constraints given by nonconvex sets.

Section 5 deals with deriving necessary optimality conditions for all the three kinds of relative Pareto minimizers induced by (1.5)-(1.7), as well as for their efficient and weak efficient counterparts, in general classes of constrained multiobjective problem. Our approach is based on reducing relative minimizers to extremal systems of sets and then using the extremal principle together with appropriate rules of generalized differential calculus as well as calculus results for sequential normal compactness properties crucial in infinite dimensions. The results obtained in the paper allow us to unify new necessary conditions for relative Pareto minimizers with improved versions of the corresponding results obtained before for the conventional notions of Pareto efficiency and weak efficiency. We discuss various particular cases and compare the new developments with some other necessary conditions in multiobjective optimization known in the literature.

Throughout the paper we use the standard notation of variational analysis; cf. [17, 21]. Unless otherwise stated, all the spaces under consideration are Banach with their norms denoted $\|\cdot\|$. Given a space $X$, we consider its topological dual $X^{*}$ equipped with the weak ${ }^{*}$ topology $w^{*}$ and use the symbols $\mathbb{B}$ and $\mathbb{B}^{*}$ for the closed unit balis in $X$ and $X^{*}$, respectively. Recall the symbol

$$
\begin{aligned}
\underset{x \rightarrow \bar{x}}{\operatorname{Limsup}} F(x):=\left\{x^{*} \in X^{*} \mid\right. & \exists \text { sequences } x_{k} \rightarrow \bar{x} \text { and } x_{k}^{*} \stackrel{w^{*}}{\rightarrow} x^{*} \\
& \text { with } \left.x_{k}^{*} \in F\left(x_{k}\right) \text { for all } k \in \mathbb{N}\right\},
\end{aligned}
$$

which stands for the sequential Painlevé-Kuratowski upper/outer limit of a set-valued mapping $F: X \rightrightarrows X^{*}$ in the norm topology of $X$ and weak* topology of $X^{*}$, where $\mathbb{N}:=\{1,2, \ldots\}$.

\section{Basic Tools of Generalized Differentiation}

In this section we define and briefly review some basic tools of generalized differentiation that are largely used in what follows to derive existence theorems and necessary optimality conditions for relative Pareto minimizers to the multiobjective optimization problems under consideration. More specific constructions and facts of generalized differentiation needed for the necessary optimality conditions are mentioned in Section 5. Except for the subdifferentials of set-valued mappings recently introduced in [3], all the details, proofs, and further discussions can be found in [17]. We also refer the reader to $[9,18,21]$ for additional material and numerous applications of these and related generalized differential constructions of variational analysis.

In what follows we present the definitions and properties of the basic generalized differential constructions held in the Asplund space setting, which is the main framework of their applications in this paper. Some useful modifications and analogs in other (including arbitrary) Banach space settings can be found in [17]. Recall that a Banach space is Asplund if each of its separable subspace has a separable dual. This remarkable class has been comprehensively investigated in geometric theory of Banach spaces and has been largely employed in variational analysis; see, e.g., 
$[9,17,18]$ and the references therein. It contains, in particular, every reflexive Banach space and every Banach space with a separable dual. In this section we assume that all the spaces under consideration are Asplund. Note that the product of Asplund spaces is Asplund as well.

Given a subset $\Omega \subset X$ closed around $\bar{x} \in \Omega$, the prenormal/Fréchet normal cone to $\Omega$ at $\ddot{x}$ is

$$
\widehat{N}(\bar{x} ; \Omega):=\left\{x^{*} \in X^{*} \mid \limsup _{x \Omega_{\bar{x}}} \frac{\left\langle x^{*}, x-x\right\rangle}{\|x-\bar{x}\|} \leq 0\right\},
$$

where the symbol $x \stackrel{\Omega}{\rightarrow} \bar{x}$ stands for $x \rightarrow \bar{x}$ with $x \in \Omega$. The (basic, limiting, Mordukhovich) normal cone to $\Omega$ at $\bar{x}$ is defined by

$$
N(\bar{x} ; \Omega):=\operatorname{Limsup}_{\substack{\Omega \rightarrow \\ x \rightarrow \bar{x}}} \hat{N}(x ; \Omega)
$$

via the sequential Painlevé-Kuratowski outer limit (1.9) of the mapping $\widehat{N}(\cdot ; \Omega): X \rightrightarrows X^{*}$. In contrast to (2.1), the basic normal cone (2.2) and the associated generalized differential constructions for mappings and functions enjoy full calculus in the framework of Asplund spaces being generally nonconvex even in finite dimensions. This calculus is largely based on the extremal principle of variational analysis; see [17, Chapters 2 and 3] for more details. For convex sets $\Omega$, both constructions (2.1) and (2.2) reduce to the normal cone of convex analysis.

Considering next a set-valued mapping $F: X \rightrightarrows Z$ with the graph

$$
\operatorname{gph} F:=\{(x, z) \in X \times Z \mid z \in F(x)\},
$$

define its Fréchet coderivative at $(\bar{x}, \bar{z}) \in \operatorname{gph} F$ by

$$
\widehat{D}^{*} F(\bar{x}, \bar{z})\left(z^{*}\right):=\left\{x^{*} \in X^{*} \mid\left(x^{*},-z^{*}\right) \in \widehat{N}((\bar{x}, \bar{z}) ; \operatorname{gph} F)\right\}
$$

and its basic/normal coderivative at $(\bar{x}, \bar{z}) \in \operatorname{gph} F$ by

$$
D^{*} F(\bar{x}, \bar{z})\left(z^{*}\right):=\left\{x^{*} \in X^{*} \mid\left(x^{*},-z^{*}\right) \in N((\bar{x}, \bar{z}) ; \operatorname{gph} F)\right\},
$$

where we omit $\bar{z}=f(\bar{x})$ if $F=f: X \rightarrow Z$ is single-valued. Observe that both coderivative set-valued mappings $\widehat{D}^{*} F(\bar{x}, \bar{z}): Z^{*} \rightrightarrows X^{*}$ and $D^{*} F(\bar{x}, \bar{z}): Z^{*} \rightrightarrows X^{*}$ are positively homogeneous reducing to the single-valued adjoint derivative linear operator

$$
\widehat{D}^{*} f(\bar{x})=\left\{\nabla f(\bar{x})^{*} z^{*}\right\}, \quad D^{*} f(\bar{x})=\left\{\nabla f(\bar{x})^{*} z^{*}\right\} \text { for all } z^{*} \in Z^{*}
$$

provided that $f$ is Fréchet differentiable at $\bar{x}$ for the first equality and strictly differentiable at this point for the second one, i.e.,

$$
\lim _{x, u \rightarrow \bar{x}} \frac{f(x)-f(u)-\langle\nabla f(\bar{x}), u-x\rangle}{\|x-u\|}=0,
$$

which is automatic when $f \in C^{1}$ around $\bar{x}$.

In this paper we are primarily dealing with set-valued and single-valued mappings whose range spaces are partially ordered. Given such a set-valued mapping $F: X \rightrightarrows Z$, assume that the order " $\leq$ " on $Z$ is generated in (1.1) by a cone $\Theta \subset Z$, which is closed, convex, and proper, i.e., $\Theta \neq\{0\}$ and $\Theta \neq Z$. Consider the epigraph of $F$ with respect to the above order by

$$
\text { epi } F:=\{(x, z) \in X \times Z \mid z \in F(x)+\Theta\}
$$


and associate with $F$ its epigraphical multifunction $\mathcal{E}_{F}: X \rightrightarrows Z$ given by

$$
\mathcal{E}_{F}(x):=\{z \in Z \mid z \in F(x)+\Theta\} \text { with } \operatorname{gph} \mathcal{E}_{F}=\operatorname{epi} F,
$$

where the ordering cone $\Theta$ is not mentioned in the epigraphical notation for simplicity. Using coderivatives of the epigraphical multifunction (2.7), we can define appropriate extensions of the subdifferential notion from extended-real-valued functions to vector-valued and set-valued mappings with values in partially ordered spaces.

The following two constructions generated by the coderivatives (2.3) and (2.4) play a crucial role in this paper. Given $(\bar{x}, \bar{z}) \in$ epi $F$, the Fréchet subdifferential of $F$ at $(\bar{x}, \bar{z})$ and the basic/normal subdifferential of $F$ at $(\bar{x}, \bar{z})$ are defined, respectively, by

$$
\begin{aligned}
& \widehat{\partial} F(\bar{x}, \bar{z}):=\left\{x^{*} \in X^{*} \mid x^{*} \in \widehat{D}^{*} \mathcal{E}_{F}(\bar{x}, \bar{z})\left(z^{*}\right), \quad-z^{*} \in N(0 ; \Theta),\left\|z^{*}\right\|=1\right\}, \\
& \partial F(\bar{x}, \bar{z}):=\left\{x^{*} \in X^{*} \mid x^{*} \in D^{*} \mathcal{E}_{F}(\bar{x}, \bar{z})\left(z^{*}\right), \quad-z^{*} \in N(0 ; \Theta),\left\|z^{*}\right\|=1\right\} .
\end{aligned}
$$

In the case of lower semicontinuous extended-real-valued functions $\varphi: X \rightarrow(-\infty, \infty]$ finite at $\bar{x}$, the subdifferential (2.8) reduces to the standard Fréchet/viscosity subdifferential of $\varphi$ at $\bar{x}$ :

$$
\widehat{\partial} \varphi(\bar{x})=\left\{x^{*} \in X^{*} \mid \liminf _{x \rightarrow \bar{x}} \frac{\varphi(x)-\varphi(\bar{x})-\left\langle x^{*}, x-\bar{x}\right\rangle}{\|x-\bar{x}\|} \geq 0\right\},
$$

while (2.9) is the (basic, limiting) subdifferential by Mordukhovich:

$$
\partial \varphi(\bar{x})=\operatorname{Limsup}_{\substack{x \rightarrow \bar{x} \\ \varphi(x) \rightarrow \varphi(\bar{x})}} \hat{\partial} \varphi(x) .
$$

Finally in this section, let us formulate the fundamental extremal principle of variational analysis [17, Theorem 2.20] for the case of two closed sets $\Omega_{1}$ and $\Omega_{2}$ in the Asplund space $X$. We say that a point $\bar{x} \in \Omega_{1} \cap \Omega_{2}$ is locally extremal for the set system $\left\{\Omega_{1}, \Omega_{2}\right\}$ if there is a neighborhood $V$ of $\tilde{x}$ such that for any $\varepsilon>0$ we can find $a \in \varepsilon B$ with

$$
\Omega_{1} \cap\left(\Omega_{2}+a\right) \cap V=\emptyset .
$$

The Extremal Principle. Let $\bar{x}$ be a local extremal point of the set system $\left\{\Omega_{1}, \Omega_{2}\right\}$, where both $\Omega_{1}$ and $\Omega_{2}$ are locally closed around $\bar{x}$. Then for every $\varepsilon>0$ there are

$$
x_{i} \in \Omega_{i} \cap(\bar{x}+\varepsilon \mathbb{B}) \text { and } x_{i}^{*} \in \widehat{N}\left(x_{i} ; \Omega_{i}\right), \quad i=1,2,
$$

satisfying the relationships

$$
1-\varepsilon \leq\left\|x_{1}^{*}\right\|+\left\|x_{2}^{*}\right\| \leq 1+\varepsilon, \quad\left\|x_{1}^{*}+x_{2}^{*}\right\| \leq \varepsilon .
$$

The extremal principle can be viewed as a nonconvex variational counterpart of the classical separation principle for convex sets. It plays in fact a fundamental role in variational analysis similar to that played by the convex separation and Bishop-Phelps theorems in the presence of convexity; see the books $[17,18]$ for more details and numerous applications. 


\section{Enhanced Variational Principles for Set-Valued Mappings}

The main goal of this section is to derive two variational principles for set-valued mappings with values in partially ordered spaces. The first result is an extension of the set-valued version [3] of the seminal Ekeland variational principle [11] and the second one extends the subdifferential variational principle established in [3] as a set-valued version of the subdifferential variational principle for scalar functions suggested by Mordukhovich and Wang [20]; see also [17, Theorem 2.28].

Let $F: X \rightrightarrows Z$ be a set-valued mapping between Banach spaces, where $Z$ is partially ordered by a proper, closed, and convex cone $\Theta \subset Z$. The major difference between the new versions of the Ekeland variational principle and the subdifferential variational principle obtained in this paper and the previous ones derived in [3] is the usage of the following limiting monotonicity condition imposed on the mapping $F$. As usual, denote the domain of $F$ by

$$
\operatorname{dom} F:=\{x \in X \mid F(x) \neq \emptyset\}
$$

and denote by Min $\Xi$ the collections of Pareto minimal/efficient points (1.2) of $\Xi \subset Z$ with respect to the ordering cone $\Theta$ on $Z$ that can be equivalently written as

$$
\operatorname{Min} \Xi:=\{\bar{z} \in \Xi \mid \bar{z}-z \notin \Theta \text { whenever } z \in \Xi, z \neq \bar{z}\} \text {. }
$$

Definition 3.1 (limiting monotonicity condition). Given $F: X \rightrightarrows Z$ and $\bar{x} \in \operatorname{dom} F$, say that $F$ satisfies the LIMITING MONOTONICITY CONDITION at $\bar{x}$ if for any sequence of pairs $\left\{\left(x_{k}, z_{k}\right)\right\} \subset \operatorname{gph} F$ with $x_{k} \rightarrow \bar{x}$ as $k \rightarrow \infty$ we have the implication:

$$
z_{k+1} \leq z_{k} \Longrightarrow \exists \ddot{z} \in \operatorname{Min} F(\bar{x}) \text { with } \bar{z} \leq z_{k}, \quad k \in \mathbb{N} .
$$

The limiting monotonicity condition (3.2) was first used in the proof of Theorem 4.1 from [3] on the existence of weak Pareto minimizers to multiobjective optimization problems with compact constraint sets. Now we are going to employ this condition in deriving the afore-mentioned variational principles. To proceed, we first need to recall the following known notions from setvalued analysis and vector optimization regarding the ordering cone $\Theta \subset Z$ and the mapping $F: X \rightrightarrows Z$ under consideration; cf. $[3,15,16,17]$ :

$-\Theta$ is pointed if $\Theta \cap(-\Theta)=\{0\}$.

$-\Theta$ has the normality property if the set $(B+\Theta) \cap(B-\Theta)$ is bounded in $Z$.

$-F$ is epiclosed if its epigraph (2.6) is closed in $X \times Z$.

$-F$ is level-closed if its $z$-level sets

$$
\mathcal{L}(z):=\{x \in X \mid \exists v \in F(x) \text { with } v \leq z\}=\{x \in X \mid F(x) \cap(z-\Theta) \neq \emptyset\}
$$

are closed in $X$ for all $z \in Z$.

$-F$ is quasibounded from below if there is a bounded subset $M \subset Z$ such that $F(X) \subset M+\Theta$. Correspondingly, a set $\Omega \subset Z$ is quasibounded from below if the constant mapping $F(x) \equiv \Omega$ enjoys this property.

$-F$ has the domination property at $\bar{x} \in \operatorname{dom} F$ if

$$
\text { for every } z \in F(x) \text { there is } v \in \operatorname{Min} F(x) \text { with } v \leq z \text {. }
$$

It is easy to see that the normality property of $\Theta$ implies its pointedness property of this cone but not vice versa. Also, every epiclosed mapping is level-closed, but the opposite may not be 
true in the case of set-valued mappings, e.g., for $F: \mathbb{R} \rightrightarrows \mathbb{R}$ given by $F(x):=0$ if $x \neq 0$ and $F(x):=(-1,1]$ if $x=0$.

Observe further that every level-closed and single-valued mapping obviously enjoys the limiting monotonicity condition (3.2). Let us present some sufficient conditions ensuring this condition for set-valued mappings. Indeed, the next proposition shows that the domination property implies the limiting monotonicity condition for broad classes of set-valued mappings $F$ and ordering cones $\Theta$.

Proposition 3.2 (sufficient conditions for limiting monotonicity). Let $F: X \rightrightarrows Z$ be level-closed, and let $\bar{x} \in \operatorname{dom} F$. Then $F$ satisfies the limiting monotonicity condition at $\bar{x}$ if it has the domination property at this point and one of the following assumptions is fulfilled:

(a) The minimum set $\operatorname{Min} F(\tilde{x})$ is compact.

(b) $F$ is quasibounded from below, $\Theta$ has the normality property, and $\Theta \cap \mathbb{B}$ is compact.

Proof. To justify the limiting monotonicity condition from Definition 3.1 under the assumptions made, take a sequence $\left\{\left(x_{k}, z_{k}\right)\right\} \subset \operatorname{gph} F$ such that $x_{k} \rightarrow \bar{x}$ as $k \rightarrow \infty$ and $z_{k+1} \leq z_{k}$ for all $k \in \mathbb{N}$ and define the sets

$$
\Lambda_{k}:=\operatorname{Min} F(\bar{x}) \cap\left(z_{k}-\Theta\right)=\left\{v \in \operatorname{Min} F(\bar{x}) \mid v \leq z_{k}\right\}, \quad k \in \mathbb{N},
$$

which are obviously closed by the closedness of and $\Theta$ and the level-closedness of $F$. Furthermore, we have the inclusions $\Lambda_{k+1} \subset \Lambda_{k}$ due to $z_{k+1} \in z_{k}-\Theta$ as $k \in \mathbb{N}$ and the convexity of $\Theta$. Let us show that $\Lambda_{k} \neq \emptyset$ for all $k \in \mathbb{N}$. Indeed, fixing $k \in \mathbb{N}$ and using the monotonicity of the sequence $\left\{z_{k}\right\}$, we get the inclusions

$$
x_{k+n} \in \mathcal{L}\left(z_{k}\right) \text { for all } n \in \mathbb{N},
$$

which imply that $\bar{x} \in \mathcal{L}\left(z_{k}\right)$ by the assumed level-closedness of $F$. Thus there is $u_{k} \in F(\bar{x})$ satisfying $u_{k} \leq z_{k}$. Employing the domination property of $F$ at $\bar{x}$, we find some $v_{k} \in F(\bar{x})$ such that $v_{k} \leq u_{k} \leq z_{k}$, which therefore justifies the desired nonemptiness $\Lambda_{k} \neq \emptyset$ for all $k \in \mathbb{N}$.

Next we prove that any sequence $\left\{v_{k}\right\} \subset \Lambda_{k}$ contains a subsequence converging to some $\bar{z} \in \operatorname{Min} F(\bar{x})$ if the assumptions made in either (a) or (b) are fulfilled. Observing that $\left\{v_{k}\right\} \subset \Lambda_{1}$ by the established set decreasing $\Lambda_{k+1} \subset \Lambda_{k}$, it remains to justify the compactness of the set $\Lambda_{1}$ under the assumptions made. It immediately follows from (a) due to the structure of $\Lambda_{1}$ in (3.4). To proceed in the case of $(b)$, observe by the assumed quasiboundedness of $F$ from below that there is a bounded set $M \subset Z$ and hence a number $m \in N$ such that

$$
\operatorname{Min} F(\bar{x}) \subset M+\Theta \subset m \mathbb{B}+\Theta .
$$

Thus it follows from the structure of $\Lambda_{1}$ in (3.4) that

$$
\Lambda_{1} \subset(m \mathbb{B}+\Theta) \cap\left(\left\|z_{1}\right\| \mathbb{B}-\Theta\right),
$$

which yields the boundedness of $\Lambda_{1}$ due to the assumed normality property of the ordering cone $\Theta$. Since the set $\Theta \cap \mathbb{B}$ is assumed to be compact in (b), we conclude by (3.4) that the boundedness of $\Lambda_{1}$ implies its compactness. The latter ensures the existence of $\bar{z} \in \operatorname{Min} F(\bar{x})$ such that

$$
\bar{z} \in \bigcap_{k=0}^{\infty} \Lambda_{k} \text { for all } k \in \mathbb{N} .
$$

This gives by (3.4) that $\bar{z} \leq z_{k}$ as $k \in \mathbb{N}$, which justifies the limiting monotonicity condition for $F$ at $\tilde{x}$ in case (b) and thus completes the proof of the proposition.

The following simple example demonstrates that the limiting monotonicity condition for $F$ may be strictly better than its domination property in a finite-dimensional setting with all the assumptions in (a) and (b) of Proposition 3.2 satisfied. 
Example 3.3 (limiting monotonicity strictly supersedes domination). Consider a setvalued mapping $F: \mathbb{R}^{2} \rightrightarrows \mathbb{R}^{2}$ defined by

$$
F(x)=F\left(x_{1}, x_{2}\right):= \begin{cases}\left(\left|x_{1}\right|,\left|x_{2}\right|\right) & \text { if } x_{1}=x_{2} \neq 0, \\ (\{0\} \times[0,-1)) \cup([-1,0] \times\{0\}) & \text { if }\left(x_{1}, x_{2}\right)=0, \\ \emptyset & \text { otherwise. }\end{cases}
$$

Taking $\Theta=\mathbb{R}_{+}^{2}$, we can easily check that the $F$ is level-closed, that the limiting monotonicity condition is satisfied at $\ddot{x}=0$, and that $\operatorname{Min} F(0)=\{(-1,0)\}$ is a compact set in $\mathbb{R}^{2}$. Furthermore, all the assumptions in (b) of Proposition 3.2 are obviously fulfilled. On the other hand, $F$ does not have the domination property at $\bar{x}=0$, since $\left(0,-\frac{1}{2}\right) \leq(-1,0)$.

In the rest of this section we utilize the limiting monotonicity condition in deriving enhanced versions of the Ekeland variational principle and the subdifferential variational principle for setvalued mappings. Let us first recall the notions of exact and approximate minimizers needed for formulations and proving these results.

Definition 3.4 (minimizers and approximate minimizers to set-valued mappings). Let $F: X \rightrightarrows Z$ take values in the Banach space $Z$ partially ordered by a proper cone $\Theta \subset Z$. Then:

(i) We say that the pair $(\bar{x}, \bar{z}) \in \operatorname{gph} F$ is a MINIMIZER to the mapping $F$ if $\bar{z}$ is a minimal point of the image set $F(X):=\bigcup_{x \in X} F(x)$, i.e.,

$$
(\bar{z}-\Theta) \cap F(X)=\{\bar{z}\} .
$$

(ii) Given $\varepsilon>0$ and $\xi \in \Theta \backslash\{0\}$, we say that the pair $(\bar{x}, \ddot{z}) \in \operatorname{gph} F$ is an APPRoxIMATE $\varepsilon \xi$-MINIMIZER to $F$ if

$$
z+\varepsilon \xi \not \leq \bar{z} \text { for all } z \in F(x) \text { with } x \neq \bar{x} \text {. }
$$

(iii) Given $\varepsilon>0$ and $\xi \in \Theta \backslash\{0\}$, we say that the pair $(\vec{x}, \bar{z}) \in \operatorname{gph} F$ is a STRICT APPROXIMATE $\varsigma \xi$-MINIMIZER to $F$ if there is a number $0<\widetilde{\varepsilon}<\varepsilon$ such that $(\vec{x}, \bar{z})$ is an approximate $\widetilde{\varepsilon} \xi$-minimizer to this mapping.

The next result provides an essential extension of the most recent version of the Ekeland variational principle for the case of set-valued mappings $F: X \Rightarrow Z$ established in [3] under the domination property of $F$ and the compactness of the minimal sets $\operatorname{Min} F(x)$. Now we replace these assumptions with the limiting monotonicity condition discussed above. Furthermore, we skip the pointedness assumption on the ordering cone $\Theta$ in [3] by choosing the initial direction $\xi$ from $\Theta \backslash(-\Theta)$, which reduces to the choice of $0 \neq \xi \in \Theta$ when $\Theta$ is pointed. The proof of the new result requires signification modification in comparison with that of [3, Theorem 3.2].

Theorem 3.5 (enhanced version of the Ekeland variational principle for set-valued mappings). Let $F: X \rightrightarrows Z$ be a set-valued mapping between Banach spaces, where $Z$ is partially ordered by a proper, closed, and convex cone $\Theta \subset Z$ with $\Theta \backslash(-\Theta) \neq \emptyset$, i.e., $\Theta$ is not a linear subspace of $Z$. Assume furthermore that $F$ is quasibounded from below, level-closed, and satisfies the limiting monotonicity condition on $\operatorname{dom} F$. Then for any $\varepsilon>0, \lambda>0, \xi \in \Theta \backslash(-\Theta)$, and $\left(x_{0}, z_{0}\right) \in \operatorname{gph} F$ there is $(\bar{x}, \bar{z}) \in \operatorname{gph} F$ satisfying

$$
\begin{gathered}
\bar{z}-z_{0}+\frac{\varepsilon}{\lambda}\left\|\bar{x}-x_{0}\right\| \xi \leq 0, \quad \bar{z} \in \operatorname{Min} F(\bar{x}), \quad \text { and } \\
z-\bar{z}+\frac{\varepsilon}{\lambda}\|x-\bar{x}\| \xi \not 0 \text { for all }(x, z) \in \operatorname{gph} F \quad \text { with }(x, z) \neq(\bar{x}, \bar{z}) .
\end{gathered}
$$


If furthermore $\left(x_{0}, z_{0}\right)$ is an approximate $\varepsilon \xi$-minimizer to $F$, then $\vec{x}$ can be chosen such that in addition to (3.6) and (3.7) we have

$$
\left\|\bar{x}-x_{0}\right\| \leq \lambda \text {. }
$$

Proof. Note first that it is sufficient to prove the theorem in the case of $\varepsilon=\lambda=1$. Indeed, the general case can be easily reduced to this special case by applying the latter to the mapping $\widetilde{F}(x):=\varepsilon^{-1} F(x)$ on the Banach space $X$ equipped with the equivalent norm $\lambda^{-1}\|\cdot\|$.

Having this in mind, introduce a set-valued mapping $T: X \times Z \rightrightarrows X$ defined by

$$
T(x, z):=\{y \in X \mid \exists v \in F(y) \text { with } v-z+\|x-y\| \xi \leq 0\}
$$

and observe that $T$ enjoys the following properties:

- The sets $T(x, z)$ are nonempty for all $z \in F(x)$, since $x \in T(x, z)$.

- The sets $T(x, z)$ are closed for all $z \in F(x)$, since the mapping $F$ is level-closed.

- The sets $T(x, z)$ are uniformly bounded for all $z \in F(x)$, since the mapping $F$ is quasibounded from below. Indeed, the latter property yields

$$
T(x, z) \subset\{y \in X \mid\|x-y\| \xi \in z-M-\Theta\} .
$$

- We have the inclusion

$$
T(y, v) \subset T(x, z) \text { if } y \in T(x, z) \text { and } v \in F(y) \text { with } v-z+\|y-x\| \xi \leq 0 .
$$

Indeed, pick $u \in T(y, v)$ and by construction of $T$ find $w \in F(u)$ satisfying

$$
w-v+\|u-y\| \xi \leq 0 .
$$

Summing the latter inequality with the one in (3.10) and taking into account that

$$
(\|x-u\|-\|u-y\|-\|y-x\|) \xi \leq 0
$$

by the triangle inequality and the choice of $\xi \in \Theta$, we have

$$
\begin{aligned}
w-z+\|x-u\| \xi & =(w-v+\|u-y\| \xi)+(v-z+\|y-x\| \xi) \\
& +(\|x-u\|-\|u-y\|-\|y-x\|) \xi \leq 0,
\end{aligned}
$$

which implies that $u \in T(x, z)$.

Now we inductively construct a sequence of pairs $\left\{\left(x_{k}, z_{k}\right)\right\} \subset \operatorname{gph} F$ by the following iterative procedure: starting with $\left(x_{0}, z_{0}\right)$ given in theorem and having the $k$-iteration $\left(x_{k}, z_{k}\right)$, we select the next one $\left(x_{k+1}, z_{k+1}\right)$ by

$$
\left\{\begin{array}{l}
x_{k+1} \in T\left(x_{k}, z_{k}\right), \\
\left\|x_{k+1}-x_{k}\right\| \geq \sup _{x \in T\left(x_{k}, z_{k}\right)}\left\|x-x_{k}\right\|-(k+1)^{-1}, \\
z_{k+1} \in F\left(x_{k+1}\right), \quad z_{k+1}-z_{k}+\left\|x_{k+1}-x_{k}\right\| \xi \leq 0,
\end{array}\right.
$$

where $k \in\{0\} \cup \mathbb{N}$. It is clear from the construction and afore-mentioned properties of $T(x, z)$ in (3.9) that the iterative procedure (3.11) is well defined. Summing up the last inequality in (3.11) from $k=0$ to $n$, we get

$$
t_{n} \xi \in z_{0}-z_{n+1}-\Theta \subset z_{0}-M-\Theta \text { with } t_{n}:=\sum_{k=0}^{n}\left\|x_{k+1}-x_{k}\right\| .
$$


Let us prove, by passing to the limit as $n \rightarrow \infty$ in (3.12) and using the assumptions made, that

$$
\sum_{k=0}^{\infty}\left\|x_{k+1}-x_{k}\right\|<\infty
$$

Arguing by contradiction, suppose that (3.13) does not hold, i.e., the increasing sequence $\left\{t_{n}\right\}$ tends to $\infty$ as $n \rightarrow \infty$. By the first inclusion in (3.12) and the boundedness of the set $M$ taken from the quasiboundedness of $F$ from below we find a bounded sequence $\left\{v_{n}\right\} \subset z_{0}-M$ satisfying

$$
t_{n} \xi-v_{n} \in-\Theta \text {, i.e., } \xi-\frac{v_{n}}{t_{n}} \in-\Theta \text { for all } n \in \mathbb{N} \text {. }
$$

Passing to the limit as $n \rightarrow \infty$ in the latter inclusion and taking into account the closedness of $\Theta$, the boundedness of $\left\{v_{n}\right\}$ and that $t_{n} \rightarrow \infty$ as $n \rightarrow \infty$, we arrive at $\xi \in-\Theta$. This contradicts the choice of $\xi \in \Theta \backslash(-\Theta)$ and thus justifies (3.13).

Further, it follows from (3.11) that $\operatorname{diam} T\left(x_{k+1}, z_{k+1}\right) \leq \operatorname{diam} T\left(x_{k}, z_{k}\right)$ and that

$$
\operatorname{diam} T\left(x_{k}, z_{k}\right) \leq 2 \sup _{x \in T\left(x_{k}, z_{k}\right)}\left\|x-x_{k}\right\| \leq 2\left(\left\|x_{k+1}-x_{k}\right\|+(k+1)^{-1}\right), \quad k \in \mathbb{N}
$$

hence $\operatorname{diam} T\left(x_{k}, z_{k}\right) \downarrow 0$ as $k \rightarrow \infty$ due to (3.13). This allows us to conclude, by the completeness of the Banach space $X$, that the sets $T\left(x_{k}, z_{k}\right)$ shrink to a singleton:

$$
\bigcap_{k=0}^{\infty} T\left(x_{k}, z_{k}\right)=\{\bar{x}\} \text { with some } \bar{x} \in \operatorname{dom} F .
$$

Note that, since $x_{k} \in \mathcal{L}\left(z_{0}\right)$ for all $k \in \mathbb{N}$ by the constructions above and since $F$ is assumed to be level-closed, we have $\bar{x} \in \mathcal{L}\left(z_{0}\right)$, which verifies that $\bar{x} \in \operatorname{dom} F$ in (3.14).

Next we justify the existence of $\bar{z} \in \operatorname{Min} F(\bar{x})$ such that the pair $(\bar{x}, \bar{z})$ satisfies the major relationships (3.6) and (3.7). Observe from the third line in (3.11) and from (3.14) that we have

$$
x_{k} \rightarrow \bar{x} \text { as } k \rightarrow \infty \text { and } z_{k} \in F\left(x_{k}\right) \text { with } z_{k+1} \leq z_{k}, \quad k \in \mathbb{N} .
$$

This ensures, by the assumed limiting monotonicity condition (3.2) for the mapping $F$ on its domain, the existence of $\bar{z} \in \operatorname{Min} F(\bar{x})$ such that $\bar{z} \leq z_{k}$ for all $k \in \mathbb{N}$. Let us prove that the pair $(\bar{x}, \bar{z}) \in \operatorname{gph} F$ satisfies the desired relationships (3.6) and (3.7).

In fact, the inclusion in (3.6) immediately follows from the choice of $\ddot{z}$. To proceed further, fix $k \in\{0\} \cup \mathbb{N}$ and sum up all the inequalities in the third line of (3.11) from $k$ to $(k+n-1)$ as $n \in \mathbb{N}$ with that of $\bar{z}-z_{k} \leq 0$. Taking into account the triangle inequality for the norm function, we get in this way that

$$
\bar{z}-z_{k}+\left\|x_{k}-x_{k+n}\right\| \xi \leq 0, \text { for all } k \in\{0\} \cup \mathbb{N} \text { and } n \in \mathbb{N} .
$$

The passage to the limit in the above inequalities with $x_{k+n} \rightarrow \bar{x}$ as $n \rightarrow . \infty$ gives

$$
\bar{z}-z_{k}+\left\|x_{k}-\bar{x}\right\| \xi \leq 0 \text { whenever } k \in\{0\} \cup \mathbb{N},
$$

which particularly justifies the inequality in (3.6), in the case of $\varepsilon=\lambda=1$ under consideration, for $k=0$. To prove (3.7) in this case, assume the contrary and thus find a pair $(x, z)$ satisfying

$$
(x, z) \in \operatorname{gph} F \text { with }(x, z) \neq(\bar{x}, \bar{z}) \text { and } z-\bar{z}+\|x-\bar{x}\| \xi \leq 0 .
$$


If $x=\bar{x}$ in (3.16), we obviously have $z \leq \bar{z}$, which contradicts the choice of $\bar{z} \in \operatorname{Min} F(\bar{x})$. If $x \neq \bar{x}$, then we have by summing up the two inequalities (3.15) and (3.16) and combining the result with the triangle inequality that

$$
z-z_{k}+\left\|x-x_{k}\right\| \xi \leq 0 \text {, i.e., } x \in T\left(x_{k}, z_{k}\right) \text { for all } k \in\{0\} \cup \mathbb{N} \text {. }
$$

This means that $x$ from (3.16) belongs to the set intersection in (3.14). Thus $x=\bar{x}$ by (3.14), which fully justifies (3.7) as $\varepsilon=\lambda=1$ and hence in the general case as well.

To complete the proof of the theorem, it remains to estimate $\left\|\bar{x}-x_{0}\right\|$ when $\left(x_{0}, z_{0}\right)$ is chosen as an approximate $\varepsilon \xi$-minimizer to $F$. Arguing by contradiction, suppose that (3.8) does not hold, i.e., $\left\|\bar{x}-x_{0}\right\|>\lambda$. Since $\vec{x} \in T\left(x_{0}, z_{0}\right)$ and $0 \leq \xi$, we have

$$
\bar{z}-z_{0}+\varepsilon \xi \leq \bar{z}-z_{0}+\frac{\varepsilon}{\lambda}\left\|\bar{x}-x_{0}\right\| \xi \leq 0
$$

and immediately observe that the latter contradicts the choice of $\left(x_{0}, z_{0}\right)$ as an approximate $\varepsilon \xi$-minimizer to $F$. Thus (3.8) holds, which completes the proof of the theorem.

Our next result is an improvement of the subdifferential variational principle for set-valued mappings established in $[3$, Theorem 3.5] as an extension of the corresponding scalar result by Mordukhovich and Wang. The new version of the subdifferential variational principle is derived under the limiting monotonicity condition imposed on the cost mapping $F: X \rightrightarrows Z$ with no pointedness assumption imposed on the ordering cone $\Theta \subset Z$ in contrast to the domination property of $F$, the compactness of the minimal sets $\operatorname{Min} F(x)$, and the pointedness requirement on $\Theta$ needed in [3]. Similarly to $\{3,17,20\}$, the proof of the subdifferential variational principle given below is based on the extremal principle presented at the end of Section 2, and hence it requires the Asplund property of the Banach spaces in question.

Theorem 3.6 (enhanced version of the subdifferential variational principle for setvalued mappings). Let $F: X \rightrightarrows Z$ be a set-valued mapping between Asplund spaces, which is epiclosed with respect to the ordering cone $\Theta \subset Z$ in addition to the assumptions of Theorem 3.5 . Then for any $\varepsilon>0, \lambda>0, \xi \in \Theta \backslash \backslash(-\Theta)$ with $\|\xi\|=1$, and a strict approximate $\varepsilon \xi$-minimizer $\left(x_{0}, z_{0}\right) \in \operatorname{gph} F$ to the mapping $F$, there is $(\bar{x}, \bar{z}) \in \operatorname{gph} F$ such that $\left\|\bar{x}-x_{0}\right\| \leq \lambda$ and

$$
\widehat{\partial} F(\bar{x}, \bar{z}) \cap \frac{\varepsilon}{\lambda} \mathbb{B}^{*} \neq \emptyset
$$

Proof. Note first that we impose the requirement $\|\xi\|=1$ in the formulation of the theorem to get a "nicer" subdifferential condition (3.17). As follows from the arguments below, condition (3.17) can be replaced by the modified subdifferential condition

$$
\widehat{\partial} F(\bar{x}, \bar{z}) \cap \frac{\varepsilon}{\lambda}\|\xi\| \mathbb{B}^{*} \neq \emptyset
$$

if $\xi$ is selected arbitrarily from $\Theta \backslash(-\Theta)$, with no change in the proof.

Since the proof of this theorem is similar to the one given in [3, Theorem 2], based on the extremal principle from Section 2 and the new version of the Ekeland variational principle from the above Theorem 3.5 , we provide only the sketch of the proof highlighting the main changes in comparison with [3], where the reader can find more details.

Take the pair $\left(x_{0}, z_{0}\right) \in$ gph $F$ from the formulation of the theorem that is a strict approximate $\varepsilon \xi$-minimizer to the mapping $F$. Thus there is a positive number $\widetilde{\varepsilon}<\varepsilon$ such that $\left(x_{0}, z_{0}\right)$ is an approximate $\widetilde{\varepsilon} \xi$-minimizer to this mapping. Put

$$
\tilde{\lambda}:=\frac{\varepsilon+\widetilde{\varepsilon}}{2} \lambda \text { with } 0<\tilde{\lambda}<\lambda
$$


and apply the enhanced Ekeland variational principle from Theorem 3.5 to the mapping $F$ and its approximate $\widetilde{\varepsilon} \xi$-minimizer $\left(x_{0}, z_{0}\right)$ with the chosen parameters $\widetilde{\varepsilon}$ and $\widetilde{\lambda}$. By (3.6)-(3.8) we find $(\bar{u}, \bar{v}) \in \operatorname{gph} F$ satisfying the relationships

$$
\begin{gathered}
\bar{v} \in \operatorname{Min} F(\overline{\bar{u}}), \quad\left\|x_{0}-\bar{u}\right\| \leq \tilde{\lambda}, \quad \text { and } \\
z-\bar{v}+\frac{\widetilde{\widetilde{\varepsilon}}}{\widetilde{\lambda}}\|x-\bar{u}\| \xi \not \leq 0 \text { for all }(x, z) \in \operatorname{gph} F \text { with }(x, z) \neq(\bar{u}, \bar{v}) .
\end{gathered}
$$

Consider a single-valued Lipschitz continuous mapping $g: X \rightarrow Z$ given by

$$
g(x):=\bar{v}-\frac{\widetilde{\varepsilon}}{\tilde{\lambda}}\|x-\bar{u}\| \xi
$$

and construct the following two closed subsets of the Asplund product space $X \times Z$ by

$$
\Omega_{1}:=\text { epi } F \text { and } \Omega_{2}:=\operatorname{gph} g \text {. }
$$

We claim that $(\bar{u}, \vec{v})$ is an extremal point of the set system $\left\{\Omega_{1}, \Omega_{2}\right\}$ from (3.22) in sense defined in Section 2. Indeed, the condition $(\bar{u}, \bar{v}) \in \Omega_{1} \cap \Omega_{2}$ is obvious, and by (2.10) we need to check the existence of a sequence $\left\{a_{k}\right\} \subset X \times Z$ such that $a_{k} \rightarrow 0$ as $k \rightarrow \infty$ and $\Omega_{1} \cap\left(\Omega_{2}+a_{k}\right)=\emptyset$ for all $k \in \mathbb{N}$. To proceed, select an arbitrary vector $\vartheta \in \Theta \backslash\{0\}$ and show that

$$
\Omega_{1} \cap\left(\Omega_{2}+\left(0,-k^{-1} \vartheta\right)\right)=\emptyset \text { for all } k \in \mathbb{N},
$$

i.e., (2.10) holds with $a_{k}=\left(0,-k^{-1} \theta\right)$. By the contrary, suppose that (3.23) does not hold for some fixed $k \in \mathbb{N}$. Then by the constructions of $\Omega_{1}$ and $\Omega_{2}$ in (3.22), there is $(x, v)$ satisfying

$$
v=g(x)-k^{-1} \vartheta \text { and }(x, v) \in \text { epi } F \text {. }
$$

Using the epigraph definition (2.6), find $z \in \operatorname{gph} F$ and $\theta \in \Theta$ with $v=z+\theta$ and substituting the latter into (3.24) we arrive at the relationships

$$
z=v-\theta=g(x)-k^{-1} \vartheta-\theta \leq g(x),
$$

since $-\vartheta \leq 0$ and $-\theta \leq 0$. Taking into account the construction of $g$ in (3.21), condition (3.20) implies now that $(x, z)=(\bar{u}, \bar{v})$. By $(3.24)$ we have therefore that

$$
v=z+\theta=\bar{v}+\theta=g(\bar{u})-k^{-1} \vartheta=\bar{v}-k^{-1} \vartheta \notin \bar{v}-\Theta,
$$

where the latter relationship holds due to $\bar{v} \in \operatorname{Min} F(\bar{u})$ in (3.19) and $v \neq \bar{v}$. This gives $\vartheta \notin \Theta$, which contradicts the above choice of $\vartheta$ and thus justifies that the reference point $(\bar{u}, \bar{v})$ is extremal for the set system (3.22).

Next let us apply to the system $\left\{\Omega_{1}, \Omega_{2},(\bar{u}, \bar{v})\right\}$ the extremal principle formulated in Section 2 imposing the sum norm $\|(x, z)\|:=\|x\|+\|z\|$ on $X \times Z$ that generates the dual norm

$$
\left\|\left(x^{*}, z^{*}\right)\right\|=\max \left\{\left\|x^{*}\right\|,\left\|z^{*}\right\|\right\} \text { for }\left(x^{*}, z^{*}\right) \in X^{*} \times Z^{*}
$$

on $X^{*} \times Z^{*}$. In this way for any $\nu>0$ we find elements $\left(x_{i}, z_{i}, x_{i}^{*}, z_{i}^{*}\right) \in X \times Z \times X^{*} \times Z^{*}$ with $i=1,2$ satisfying the relationships

$$
\left\{\begin{array}{l}
\left(x_{i}, z_{i}\right) \in \Omega_{1} \times \Omega_{2}, \quad\left\|x_{i}-\bar{u}\right\|+\left\|z_{i}-\bar{v}\right\| \leq \nu, \quad i=1,2 \\
\left(x_{i}^{*},-z_{i}^{*}\right) \in \widehat{N}\left(\left(x_{i}, z_{i}\right) ; \Omega_{i}\right), \quad i=1,2, \\
\frac{1}{2}-\nu \leq \max \left\{\left\|x_{i}^{*}\right\|,\left\|z_{i}^{*}\right\|\right\} \leq \frac{1}{2}+\nu, \quad i=1,2 \\
\max \left\{\left\|x_{1}^{*}+x_{2}^{*}\right\|,\left\|z_{1}^{*}+z_{2}^{*}\right\|\right\} \leq \nu
\end{array}\right.
$$


It follows from the Lipschitz continuity of the mapping $g$ in (3.21) with constant $\ell=\tilde{\varepsilon} / \tilde{\lambda}$ and the coderivative estimate from [17, Theorem 1.43$]$ that

$$
\left\|x_{2}^{*}\right\| \leq \frac{\widetilde{\varepsilon}}{\tilde{\lambda}}\left\|z_{2}^{*}\right\| \text { and hence } z_{2}^{*} \neq 0
$$

by (3.25) with $\nu>0$ sufficiently small. The latter yields by the extremal principle (3.25) that

$$
\left\|z_{1}^{*}\right\| \neq 0 \text { and } \frac{\left\|x_{1}^{*}\right\|}{\left\|z_{1}^{*}\right\|}<\frac{\varepsilon}{\lambda}
$$

see more details in the similar setting of [3, Theorem 3.5]. Furthermore, from the second line in (3.25) with $i=1$ we find $\widetilde{z}_{1} \in F\left(x_{1}\right)$ with

$$
\left(x_{1}, \tilde{z}_{1}\right) \in \operatorname{gph} F, \quad\left(x_{1}^{*},-z_{1}^{*}\right) \in \widehat{N}\left(\left(x_{1}, \tilde{z}_{1}\right) ; \text { epi } F\right), \text { and }-z_{1}^{*} \in \widehat{N}(0 ; \Theta) .
$$

Denoting finally $(\bar{x}, \bar{z}):=\left(x_{1}, \widetilde{z}_{1}\right), x^{*}:=x_{1}^{*} /\left\|z_{1}^{*}\right\|$, and $z^{*}:=z_{1}^{*} /\left\|z_{1}^{*}\right\|$ and taking into account the Fréchet subdifferential construction (2.8), we get the desired subdifferential condition (3.17) from the relationships in (3.26). To complete the proof of the theorem, it remains to observe that the estimate $\left\|\bar{x}-x_{0}\right\| \leq \lambda$ follows from the second inequality in (3.19), the first line in (3.25) for $i=1$, and the choice of $\tilde{\lambda}$ in (3.18).

\section{Existence of Relative Pareto Minimizers}

This section is devoted to deriving verifiable conditions that ensure the existence of relaxed Pareto minimizers to general multiobjective problems. Our primary multiobjective problem is minimizing a set-valued mapping $F: X \rightrightarrows Z$ with values in a partially ordered space. This includes the case of $F(x)=\emptyset$ for some $x \in X$ and thus implicitly involves the constraints $x \in \operatorname{dom} F$. On the other hand, explicit constraints the type $x \in \Omega$ and their specifications can be reduced to minimizing such mappings by imposing $F(x)=\emptyset$ for $x \notin \Omega$. We employ this device to study the existence issues in constrained multiobjective optimization at the end of this section.

Let us first define the new notions of relaxed minimizers to set-valued mappings $F: X \rightrightarrows Z$ with $\operatorname{dom} F \neq \emptyset$ studied in this and next sections. These notions are generated by the corresponding definitions of relative/intrinsic relative/quasi relative interior minimal points of sets from Section 1 . Observe that they are defined similarly to weak Pareto minimizers to $F$, which are those $(\bar{x}, \bar{z}) \in$ gph $F$ satisfying

$$
(\bar{z}-\operatorname{int} \Theta) \cap F(X)=\emptyset \text { provided that } \operatorname{int} \Theta \neq \emptyset .
$$

Recall that our standing assumptions imposed on the ordering cone $\Theta \subset Z$ is that $\Theta$ is proper, close, and convex (while may not be pointed) in the Banach space $Z$.

Definition 4.1 (relative Pareto minimizers to multiobjective problems). Given a mapping $F: X \rightrightarrows Z$ with the range space $Z$ partially ordered by a cone $\Theta \subset Z$, we say that:

(i) $(\bar{x}, \bar{z}) \in \operatorname{gph} F$ is a (PRIMARY) RELATIVE MINIMIZER to $F$ if

$$
(\bar{z}-\operatorname{ri} \Theta) \cap F(X)=\emptyset \text { provided that } \operatorname{ri} \Theta \neq \emptyset \text {. }
$$

(ii) $(\bar{x}, \bar{z}) \in$ gph $F$ is an INTRINSIC RELATIVE MINIMIZER to $F$ if

$$
(\bar{z}-\operatorname{iri} \Theta) \cap F(X)=\emptyset \text { provided that } \operatorname{iri} \Theta \neq \emptyset \text {. }
$$

(iii) $(\bar{x}, \bar{z}) \in \operatorname{gph} F$ is a QUASI RELATIVE MINIMIZER to $F$ if

$$
(\bar{z}-\text { qri } \Theta) \cap F(X)=\emptyset \text { provided that } \text { qri } \Theta \neq \emptyset .
$$


According to the discussions in Section 1, all the three relative Pareto minimizers to $F$ from Definition 4.1 agree if $\mathrm{ri} \Theta \neq \emptyset$. Furthermore, they all are weak Pareto minimizers (4.1) when $\operatorname{int} \Theta \neq \emptyset$. At the same time, all the relative minimizers (4.2)-(4.4) make sense when int $\Theta=\emptyset$, and both intrinsic relative minimizers (4.3) and quasi relative minimizers (4.4) make sense when ri $\Theta=\emptyset$. Recall that all the relative Pareto minimizers from Definition 4.1 are surely well defined, in contrast to weak Pareto minimizers (4.1), when the space $Z$ is finite-dimensional while quasi relative minimizers (4.4) are well defined in every separable spaces $Z$ for any ordering cones $\Theta \subset Z$.

The main result of this section ensures the existence of intrinsic relative minimizers (4.3) provided that iri $\Theta \neq \emptyset$, and hence the existence of primary relative minimizers (4.2) provided that ri $\Theta \neq \emptyset$, under verifiable conditions involving subdifferentials of set-valued mappings. Note that the result obtained below establishes a new existence theorem even in the case of weak Pareto minimizers (4.1) with int $\Theta \neq \emptyset$ for general set-valued mappings between Asplund spaces. In the next section we derive pointwise necessary optimality conditions for all the types of (localized) relative Pareto minimizers introduced in Definition 4.1 as well as for their Pareto/efficient and weak Pareto counterparts.

The principal condition ensuring the existence of weak minimizers to mappings $F: X \rightrightarrows Z$ between Asplund spaces in our previous work [3] was the following basic subdifferential PalaisSmale condition: every sequence $\left\{x_{k}\right\} \subset \operatorname{dom} F$ such that

$$
\text { there are } z_{k} \in F\left(x_{k}\right) \text { and } x_{k}^{*} \in \partial F\left(x_{k}, z_{k}\right) \text { with }\left\|x_{k}^{*}\right\| \rightarrow 0 \text { as } k \rightarrow \infty
$$

contains a convergent subsequence, provided that $\left\{z_{k}\right\} \subset Z$ is quasibounded from below. In (4.5), we use the basic/normal subdifferential of $F$ at $\left(x_{k}, z_{k}\right)$ defined in $(2.9)$. In the case of smooth around $x_{k}$, or strictly differentiable at $x_{k}$, real-valued functions $F=\varphi: X \rightarrow \mathbb{R}$, the subdifferential Palais-Smale condition (4.5) reduces to the classical one due to $\partial \varphi\left(x_{k}\right)=\left\{\nabla \varphi\left(x_{k}\right)\right\}, k \in \mathbb{N}$, in this case; see the second formula in (2.5).

In this paper we introduce and employ a new version of the subdifferential Palais-Smale condition involving the Fréchet subdifferential (2.8) of $F$ instead of the normal one as in (4.5).

Definition 4.2 (refined subdifferential Palais-Smale condition for set-valued mappings). A set-valued mapping $F: X \Rightarrow Z$ satisfies the ReFined subdifferential Palais-SMALE CONDITION if every sequence $\left\{x_{k}\right\} \subset \operatorname{dom} F$ such that

$$
\text { there are } z_{k} \in F\left(x_{k}\right) \text { and } x_{k}^{*} \in \widehat{\partial} F\left(x_{k}, z_{k}\right) \text { with }\left\|x_{k}^{*}\right\| \rightarrow 0 \text { as } k \rightarrow \infty
$$

contains a convergent subsequence, provided that $\left\{z_{k}\right\} \subset Z$ is quasibounded from below.

Since we always have $\widehat{\partial} F(\bar{x}, \bar{z}) \subset \partial F(\bar{x}, \bar{z})$, the refined Palais-Smale condition (4.6) improves the previous one (4.5). Furthermore, the refined Palais-Smale condition (4.6) reduces to the classical one for functions $\varphi: X \rightarrow \mathbb{R}$ merely Fréchet differentiable at $x_{k}$, in contrast to the required strict differentiability of $\varphi$ in the case of (4.5); cf. the two formulas in (2.5).

The following major result ensures the existence of intrinsic relative minimizers to set-valued mappings under the refined subdifferential Palais-Smale (4.6) and the limiting monotonicity (3.2) conditions imposed on the mapping under consideration. Its proof (which is significantly more involved in comparison with [3, Theorem 4.3]) employs all the three variational principles derived and discussed above: the enhanced versions of the Ekeland variational principle and the subdifferential variational principle for set-valued mappings established in Theorem 3.5 and Theorem 3.6, respectively, as well as the extremal principle for systems of closed sets in Asplund spaces formulated in Section 2, which is used in the proof below in the equivalent form of the fuzzy intersection rule for Fréchet normals from [17, Lemma 3.1]. 
Theorem 4.3 (existence of intrinsic relative minimizers to set-valued mappings). Let $F: X \rightrightarrows Z$ be a mapping between Asplund spaces that is epiclosed, quasibounded from below, and satisfies the limiting monotonicity condition on $\operatorname{dom} F$. Assume furthermore that the refined subdifferential Palais-Smale condition from Definition 4.2 holds and that $\Theta \backslash(-\Theta) \neq \emptyset$, i.e., $\Theta$ is not a linear subspace of $Z$. Then $F$ admits an intrinsic relative minimizer provided that iri $\Theta \neq \emptyset$.

Proof. To justify the existence of intrinsic relaxed minimizers to $F$, we first inductively apply the new version of the Ekeland variational principle for set-valued mappings to generate a sequence $\left\{\left(x_{k}, z_{k}\right)\right\} \subset \operatorname{gph} F, k \in \mathbb{N}$. Then we prove that the chosen sequence $\left\{x_{k}\right\}$ contains a subsequence converging to an intrinsic relative minimizer to $F$. The latter arguments are rather involved based on applying the above version of the subdifferential variational principle, the refined version of the subdifferential Palais-Smale condition, the afore-mentioned fuzzy intersection description of the extremal principle, and the liniting monotonicity condition. Details follow.

To begin with, pick an arbitrary pair $\left(x_{0}, z_{0}\right) \in \operatorname{gph} F$ and element $\xi \in \Theta \backslash(-\Theta)$ with $\|\xi\|=1$ and then inductively generate a sequence $\left\{\left(x_{k}, z_{k}\right)\right\} \subset \operatorname{gph} F$ by using the set-valued version of the Ekeland variational principle from Theorem 3.5. To proceed, we fix $k \in \mathbb{N}$ and having the $(k-1)$-iteration $\left(x_{k-1}, z_{k-1}\right)$, apply Theorem 3.5 with the parameters $\varepsilon=k^{-2}$ and $\lambda=k^{-1}$ to get the next iteration $\left(x_{k}, z_{k}\right) \in \operatorname{gph} F$ satisfying the relationships

$$
\begin{gathered}
z_{k} \in \operatorname{Min} F\left(x_{k}\right), \quad z_{k} \leq z_{k-1}, \quad \text { and } \\
z-z_{k}+k^{-1}\left\|x-x_{k}\right\| \xi \leq 0 \text { for all }(x, z) \in \operatorname{gph} F \text { with }(x, z) \neq\left(x_{k}, z_{k}\right), \quad k \in \mathbb{N} .
\end{gathered}
$$

Suppose now that the chosen sequence $\left\{x_{k}\right\}$ contains a subsequence converging to some point $\tilde{x} \in \operatorname{dom} F$; we show that it is the case a bit later. Without loss of generality, assume that $x_{k} \rightarrow \bar{x}$ as $k \rightarrow \infty$ for the whole sequence $\left\{x_{k}\right\}$ and get from (4.7) and the limiting monotonicity condition of Definition 3.1 that

$$
\text { there is } \ddot{z} \in F(\bar{x}) \text { with } \bar{z} \leq z_{k} \text { for all } k \in \mathbb{N} \text {. }
$$

Let us prove that the pair $(\bar{x}, \bar{z})$ is an intrinsic relative minimizer to $F$. Indeed, taking an arbitrary pair $(x, z) \in \operatorname{gph} F$ with $(x, z) \neq(\bar{x}, \bar{z})$ and employing (4.8) and (4.9), we have by elementary transformations that

$$
z-\bar{z}+k^{-1}\left\|x-x_{k}\right\| \xi \in z_{k}-\bar{z}+Z \backslash(-\Theta) \text { for all } k \in \mathbb{N},
$$

which easily implies the inclusion

$$
z-\bar{z}+k^{-1}\left\|x-x_{k}\right\| \xi \in \Theta+Z \backslash(-\Theta)
$$

The latter gives, by the convexity of the ordering cone $\Theta$, that

$$
z-\bar{z}+k^{-1}\left\|x-x_{k}\right\| \xi \in Z \backslash(-\Theta), \quad k \in \mathbb{N} .
$$

Our next goal is the show, by passing to the limit in (4.10) as $k \rightarrow \infty$, that

$$
z-\bar{z} \in Z \backslash(-\operatorname{iri} \Theta) \text { provided that } \operatorname{iri} \Theta \neq \emptyset
$$

Arguing by contradiction, suppose that (4.11) does not hold, i.e., $z-\bar{z}=: \theta \in-$ iri $\Theta$. Employing the definition of intrinsic relative interior, we have that the cone

$$
\text { cone }(\Theta+\theta) \text { is a linear subspace of } Z \text {. }
$$


Since $\xi \in \Theta$ and $\xi+\theta \in$ cone $(\Theta+\theta)$, we get from (4.12) that $-\xi-\theta \in$ cone $(\Theta+\theta)$ as well. Taking now into account the definition of the cone generated by a set and the convexity of $\Theta$, we find a positive number $\vec{t} \leq 1$ such that

$$
t(-\xi-\theta) \in \Theta+\theta \text { for all } t \in[0, \hat{t}] .
$$

Since $\Theta$ is a cone, the last inclusion implies that

$$
\theta+\tau \xi \in-\Theta \text { for all } \tau=\frac{t}{1+t} \in\left[0, \frac{\bar{t}}{1+\vec{t}}\right]
$$

Note that $k^{-1}\left\|x-x_{k}\right\| \rightarrow 0$ as $k \rightarrow \infty$, and so we have $k^{-1}\left\|x-x_{k}\right\| \in[0, \bar{t} /(1+\bar{t})]$ for $k$ sufficient large. Substituting this into (4.13) and observing that $\theta=z-\bar{z}$ by definition, we arrive at

$$
z-\bar{z}+k^{-1}\left\|x-x_{k}\right\| \xi \in-\Theta
$$

which contradicts (4.10) and therefore justifies the inclusion (4.11). Since the pair $(x, z) \in \operatorname{gph} F$ was chosen arbitrarily in the arguments above, relationships (4.11) clearly implies that of (4.3) and thus justifies the intrinsic relative minimality of $(\bar{x}, \bar{z})$ to the mapping $F$.

To complete the proof, it remains to justify the claim announced above: the chosen sequence $\left\{x_{k}\right\}$ contains a convergent subsequence. To prove this convergence, we inductively construct another sequence $\left\{\widetilde{x}_{k}\right\} \subset \operatorname{dom} F$ such that $\left\|\widetilde{x}_{k}-x_{k}\right\| \rightarrow 0$ as $k \rightarrow \infty$ and that the refined PalaisSmale condition (4.6) can be applied to this new sequence. To proceed, define for each $k \in \mathbb{N}$ a set-valued mapping $F_{k}: X \rightrightarrows Z$ given by

$$
F_{k}(x):=F(x)+g_{k}(x) \text { with } g_{k}(x):=k^{-1}\left\|x-x_{k}\right\| \xi
$$

and conclude from (4.8) that $\left(x_{k}, z_{k}\right)$ is a strict approximate $k^{-2} \xi$-minimizer to $F_{k}$. Fix $k \in \mathbb{N}$ and apply the subdifferential variational principle for set-valued mappings from Theorem 3.6 to the mapping $F_{k}$ in (4.14) and its strict approximate $\varepsilon \xi$-minimizer $\left(x_{k}, z_{k}\right)$ with $\varepsilon=k^{-2}$ and $\lambda=k^{-1}$. Taking into account the structure of $F_{k}$ in (4.14) and the subdifferential construction (2.8), we find $\left(\widetilde{x}_{k}, \widetilde{z}_{k}, \widetilde{v}_{k}, \widetilde{x}_{k}^{*}, \widetilde{z}_{k}^{*}\right) \in X \times Z \times Z \times X^{*} \times Z^{*}$ satisfying the following relationships:

$$
\left\{\begin{array}{l}
\dot{\widetilde{z}}_{k} \in F\left(\widetilde{x}_{k}\right), \quad \widetilde{v}_{k}=g_{k}\left(\widetilde{x}_{k}\right), \quad\left(x_{k}, \widetilde{z}_{k}+\widetilde{v}_{k}\right) \in \operatorname{gph} F_{k}, \quad\left\|\widetilde{x}_{k}-x_{k}\right\| \leq k^{-1}, \\
\left(\widetilde{x}_{k}^{*},-\widetilde{z}_{k}^{*}\right) \in \widehat{N}\left(\left(\widetilde{x}_{k}, \widetilde{z}_{k}+\widetilde{v}_{k}\right) ; \operatorname{epi} F_{k}\right), \quad-\widetilde{z}_{k}^{*} \in \widehat{N}(0 ; \Theta), \quad\left\|\widetilde{z}_{k}^{*}\right\|=1, \quad\left\|\widetilde{x}_{k}^{*}\right\| \leq k^{-1} .
\end{array}\right.
$$

Consider now the Asplund product space $X \times Z \times Z$ equipped with the usual sum norm on the product (and hence by the corresponding maximum on the dual space $X^{*} \times Z^{*} \times Z^{*}$ ) and form the following two subsets of the product space:

$$
\begin{aligned}
& \Omega_{1}:=\{(x, z, v) \in X \times Z \times Z \mid(x, z) \in \operatorname{epi} F\} \\
& \Omega_{2}:=\left\{(x, z, v) \in X \times Z \times Z \mid(x, v) \in \operatorname{gph} g_{k}\right\} .
\end{aligned}
$$

It is easy to see that $\left(\widetilde{x}_{k}, \widetilde{z}_{k}, \widetilde{v}_{k}\right) \in \Omega_{1} \cap \Omega_{2}$ and that both sets $\Omega_{1}$ and $\Omega_{2}$ are locally closed around this point by the epiclosedness of $F$ and the Lipschitz continuity of $g_{k}$. Observe also that

$$
(x, z, v) \in \Omega_{1} \cap \Omega_{2} \Longrightarrow z \in F(x)+\Theta, v=g_{k}(x),
$$


and thus $(x, z+v) \in$ epi $F_{k}$. We have furthermore from the second line of (4.15) that

$$
\begin{aligned}
& \limsup _{\substack{(x, z, v) \rightarrow\left(\tilde{x}_{k}, \widetilde{z}_{k}, \tilde{v}_{k}\right) \\
(x, z, v) \in \Omega_{1} \cap \Omega_{2}}} \frac{\left\langle\left(\widetilde{x}_{k}^{*},-\widetilde{z}_{k}^{*},-\widetilde{z}_{k}^{*}\right),(x, z, v)-\left(\widetilde{x}_{k}, \widetilde{z}_{k}, \widetilde{v}_{k}\right)\right\rangle}{\left\|(x, z, v)-\left(\widetilde{x}_{k}, \widetilde{z}_{k}, \widetilde{v}_{k}\right)\right\|} \\
& \leq \limsup _{\substack{(x, z) \rightarrow\left(\widetilde{x}_{k}, \widetilde{z}_{k}+\widetilde{v}_{k}\right) \\
(x, z) \in \text { epi } F_{k}}} \frac{\left.\left\langle\widetilde{x}_{k}^{*},-\widetilde{z}_{k}^{*}\right),(x, z)-\left(\widetilde{x}_{k}, \widetilde{z}_{k}+\widetilde{v}_{k}\right)\right\rangle}{\left\|(x, z)-\left(\widetilde{x}_{k}, \widetilde{z}_{k}+\widetilde{v}_{k}\right)\right\|} \leq 0,
\end{aligned}
$$

which implies the inclusion

$$
\left(\widetilde{x}_{k}^{*},-\widetilde{z}_{k}^{*},-\widetilde{z}_{k}^{*}\right) \in \widehat{N}\left(\left(\widetilde{x}_{k}, \widetilde{z}_{k}, \widetilde{v}_{k}\right) ; \Omega_{1} \cap \Omega_{2}\right), \quad k \in \mathbb{N} .
$$

Next we are going to express Fréchet normals to the intersection $\Omega_{1} \cap \Omega_{2}$ in (4.18) via Fréchet normals to $\Omega_{1}$ and $\Omega_{2}$ and then, by taking into account the structures of these sets, to arrive at the desired conclusions in terms of the mapping $F$ under consideration. Applying to the set intersection $\Omega_{1} \cap \Omega_{2}$ the fuzzy intersection rule from [17, Lemma 3.1] equivalent to the extremal principle and using the particular structures of the sets $\Omega_{1}$ and $\Omega_{2}$ in (4.16) and (4.17), we find $t \geq 0,\left(x_{i k}, z_{i k}, v_{i k}\right) \in \Omega_{i}$, and $\left(x_{i k}^{*}, z_{i k}^{*}, v_{i k}^{*}\right) \in X^{*} \times Z^{*} \times Z^{*}$ for $i=1,2$ satisfying the relationships

$$
\left\{\begin{array}{l}
\left(x_{1}, z_{1}\right) \in \text { epi } F, \quad v_{2}=g_{k}\left(x_{2}\right), \quad\left\|x_{1}-\widetilde{x}_{k}\right\| \leq k^{-1}, \\
\left(x_{1}^{*},-z_{1}^{*}\right) \in \hat{N}\left(\left(x_{1}, z_{1}\right) ; \text { epi } F\right), \quad-z_{1}^{*} \in N(0 ; \Theta), \quad x_{2}^{*} \in \widehat{D}^{*} g_{k}\left(x_{2}\right)\left(z_{2}^{*}\right), \\
\left\|t \widetilde{x}_{k}^{*}-x_{1}^{*}-x_{2}^{*}\right\| \leq k^{-1}, \quad\left\|t \widetilde{z}_{k}^{*}-z_{1}^{*}\right\| \leq k^{-1}, \quad\left\|t \widetilde{z}_{k}^{*}-z_{2}^{*}\right\| \leq k^{-1}, \\
1-k^{-1} \leq \max \left\{t,\left\|\left(x_{2}^{*}, 0, z_{2}^{*}\right)\right\|\right\} \leq 1+k^{-1},
\end{array}\right.
$$

where for the time being we $d r o p$ the index " $k$ " in the above $i$-sequences to simplify the notation.

Working with (4.19), we first observe that $t$ must be nonzero therein. Arguing by contradiction, suppose that it is not the case, i.e., $t=0$. Then it follows from the third line of (4.19) that $\left\|z_{2}^{*}\right\| \leq k^{-1}$. Taking now into account that the mapping $g_{k}$ in (4.14) is Lipschitz continuous with modulus $k^{-1}$ and employing the coderivative estimate for Lipschitzian mappings from [17, Theorem 1.43], we get from the second line of (4.19) that

$$
\left\|x_{2}^{*}\right\| \leq k^{-1}\left\|z_{2}^{*}\right\|
$$

and therefore $\left\|x_{2}^{*}\right\| \leq k^{-2}$. This contradicts the nontriviality condition on $\left(x_{2}^{*}, 0, z_{2}^{*}\right)$ in the last line of (4.19) and thus justifies that $t>0$.

To proceed further, we consider the following two possibilities of realizing the maximum of the expression $\left\{t,\left\|\left(x_{2}^{*}, 0, z_{2}^{*}\right)\right\|\right\}$ in (4.19):

Case 1. If $\max \left\{t,\left\|\left(x_{2}^{*}, 0, z_{2}^{*}\right)\right\|\right\}=t$, then the the last line in (4.19) becomes

$$
1-k^{-1} \leq t \leq 1+k^{-1} \text {. }
$$

Substituting the upper and lower bounds of $t$ from the above estimates into the inequalities in the third line of (4.19) and taking into account the triangle inequality, estimate (4.20), and that $\left\|\widetilde{z}_{k}^{*}\right\|=1$ while $\left\|\widetilde{x}_{k}^{*}\right\| \leq k^{-1}$ in $(4.15)$, we arrive at the relationships

$$
1-2 k^{-1} \leq\left\|z_{i}^{*}\right\| \leq 1+2 k^{-1} \text { for } i=1,2 \text { and hence }
$$




$$
\begin{aligned}
\frac{\left\|x_{1}^{*}\right\|}{\left\|z_{1}^{*}\right\|} & \leq \frac{\left(t\left\|\widetilde{x}_{k}^{*}\right\|+\left\|x_{2}^{*}\right\|+k^{-1}\right)}{\left\|z_{1}^{*}\right\|} \leq \frac{\left(\left(1+k^{-1}\right) k^{-1}+k^{-1}\left(1+2 k^{-1}\right)+k^{-1}\right)}{\left(1-2 k^{-1}\right)} \\
& =\frac{3 k^{-1}+3 k^{-2}}{1-2 k^{-1}}
\end{aligned}
$$

Case 2. Assuming now that

$$
\max \left\{t,\left\|\left(x_{2}^{*}, 0, z_{2}^{*}\right)\right\|\right\}=\left\|\left(x_{2}^{*}, 0, z_{2}^{*}\right)\right\|
$$

and using that $\left\|\left(x_{2}^{*}, 0, z_{2}^{*}\right)\right\|=\left\|z_{2}^{*}\right\|$ by estimate (4.20) and the "maximum" dual norm on the product $X^{*} \times Z^{*} \times Z^{*}$, we get from the last line of (4.19) the lower and upper estimates for $\left\|z_{2}^{*}\right\|$ :

$$
1-k^{-1} \leq\left\|z_{2}^{*}\right\| \leq 1+k^{-1} \text {. }
$$

Substituting these estimates into $\left\|t \widetilde{z}_{k}^{*}-z_{1}^{*}\right\| \leq k^{-1}$ from (4.19) and taking into account that $\left\|\tilde{z}_{k}^{*}\right\|=1$ by (4.15), we obtain the lower and upper estimates for $t$ :

$$
t \geq\left\|z_{2}^{*}\right\|-k^{-1} \geq 1-2 k^{-1} \text { and } t \leq\left\|z_{2}^{*}\right\|+k^{-1} \leq 1+2 k^{-1} .
$$

Then the third line of (4.19) and the lower estimate of $t$ in (4.23) yield that

$$
\left\|z_{1}^{*}\right\| \geq t-k^{-1} \geq 1-3 k^{-1} .
$$

Let us finally estimate the ratio $\left\|x_{1}^{*}\right\| /\left\|z_{1}^{*}\right\|$. Using the inequality $\left\|t \widetilde{x}_{k}^{*}-x_{1}^{*}-x_{2}^{*}\right\| \leq k^{-1}$ from the third line of (4.19) and the one $\left\|\widetilde{x}_{k}^{*}\right\| \leq k^{-1}$ from (4.15) together with (4.20), the upper bound for $t$ in (4.23), and the lower bound of $\left\|z_{1}^{*}\right\|$ in (4.24), we get

$$
\begin{aligned}
\frac{\left\|x_{1}^{*}\right\|}{\left\|z_{1}^{*}\right\|} & \leq \frac{\left(t\left\|\widetilde{x}_{k}^{*}\right\|+\left\|x_{2}^{*}\right\|+k^{-1}\right)}{\left\|z_{1}^{*}\right\|} \leq \frac{\left(\left(1+2 k^{-1}\right) k^{-1}+k^{-1}\left(1+k^{-1}\right)+k^{-1}\right)}{1-3 k^{-1}} \\
& =\frac{3 k^{-1}\left(1+k^{-1}\right)}{1-3 k^{-1}}
\end{aligned}
$$

which ends our consideration in Case 2. Thus in both Case 1 and Case 2 we have similar (while different) estimates of the the ratio $\left\|x_{1}^{*}\right\| /\left\|z_{1}^{*}\right\|$ in (4.21) and (4.25), respectively.

Continuing now the proof of the theorem simultaneously for the above cases of realizing the maximum in the last line of (4.19), we denote

$$
\widetilde{x}_{1}^{*}:=\frac{x_{1}^{*}}{\left\|z_{1}^{*}\right\|} \text { and } \tilde{z}_{1}^{*}:=\frac{z_{1}^{*}}{\left\|z_{1}^{*}\right\|} \text { with }\left\|\tilde{z}_{1}^{*}\right\|=1
$$

and, by the first two lines in (4.19) concerning $\left(x_{1}, z_{1}, x_{1}^{*}, z_{1}^{*}\right)$ and by definition (2.8) of the Fréchet subdifferential of $F$, have

$$
\widetilde{x}_{1}^{*} \in \widehat{\partial} F\left(x_{1}, z_{1}\right) \text { with }\left(x_{1}, z_{1}\right) \in \text { epi } F .
$$

Let us show that we can get relationships similar to (4.27) while replacing $\left(x_{1}, z_{1}\right) \in$ epi $F$ by some $\left(x_{1}, \widetilde{z}_{1}\right) \in \mathrm{gph} F$, i.e., we can find $\widetilde{z}_{1}$ such that

$$
\widetilde{x}_{1}^{*} \in \widehat{\partial} F\left(x_{1}, \widetilde{z}_{1}\right) \text { with }\left(x_{1}, \widetilde{z}_{1}\right) \in \operatorname{gph} F .
$$


The latter is needed for the subsequent application of the refined subdifferential Palais-Smale condition from Definition 4.2, which deals with points of the graph.

To proceed, we write (4.27) in the normal cone form:

$$
\left(\widetilde{x}_{1}^{*},-\widetilde{z}_{1}^{*}\right) \in \widehat{N}\left(\left(x_{1}, z_{1}\right) ; \text { epi } F\right) \text { with }-\widetilde{z}_{1}^{*} \in N(0 ; \Theta), \quad\left\|\widetilde{z}_{1}^{*}\right\|=1
$$

and observe by definition (2.1) that for any $\gamma>0$ there is $\eta>0$ such that

$$
\left\langle\left(\widetilde{x}_{1}^{*},-\widetilde{z}_{1}^{*}\right),(x, z)-\left(x_{1}, z_{1}\right)\right\rangle \leq \gamma\left\|(x, z)-\left(x_{1}, z_{1}\right)\right\|
$$

for all $(x, z) \in$ epi $F$ with $x \in x_{1}+\eta \mathbb{B}$ and $z \in z_{1}+\eta \mathbb{B}$. By definition (2.6) of epi $F$ with respect to the ordering cone $\Theta$, we have

$$
z_{1} \in \widetilde{z}_{1}+\theta \text { for some } \tilde{z}_{1} \in F\left(x_{1}\right) \text { and } \theta \in \Theta .
$$

Taking further an arbitrary vector $(u, v) \in$ epi $F$ with $u \in x_{1}+\eta \mathbb{B}$ and $v \in \widetilde{z}_{1}+\eta \mathbb{B}$, observe by the above ordering that

$$
v=\widetilde{v}+\tilde{\theta} \text { for some } \widetilde{v} \in F(u) \text { and } \tilde{\theta} \in \Theta .
$$

Consider now a vector $\tilde{z} \in Z$ defined by

$$
\widetilde{z}:=v+\left(z_{1}-\widetilde{z}_{1}\right)
$$

and derive from (4.30) and (4.31) and from the convexity of the cone $\Theta$ that

$$
[\widetilde{z}=\tilde{v}+\tilde{\theta}+\theta, \quad \tilde{v} \in F(u)] \Longrightarrow(u, \widetilde{z}) \in \operatorname{epi} F .
$$

Since $\left\|\widetilde{z}-z_{1}\right\|=\left\|v-\widetilde{z}_{1}\right\| \leq \eta$ by the choice of $\widetilde{z}$, we have $(u, \widetilde{z}) \in$ epi $F$ with $u \in x_{1}+\eta \mathbb{B}$ and $\widetilde{z} \in z_{1}+\eta \mathbb{B}$. Substituting $(u, \widetilde{z})$ into (4.29) gives

$$
\left\langle\left(\widetilde{x}_{1}^{*},-\widetilde{z}_{1}^{*}\right),(u, \widetilde{z})-\left(x_{1}, z_{1}\right)\right\rangle \leq \gamma\left\|(u, \widetilde{z})-\left(x_{1}, z_{1}\right)\right\|,
$$

and hence, by $\widetilde{z}-z_{1}=v-\widetilde{z}_{1}$, we get that

$$
\left\langle\left(\widetilde{x}_{1}^{*},-\widetilde{z}_{1}^{*}\right),(u, v)-\left(x_{1}, \widetilde{z}_{1}\right)\right\rangle \leq \gamma\left\|(u, v)-\left(x_{1}, \widetilde{z}_{1}\right)\right\|,
$$

which implies $\left(\widetilde{x}_{1}^{*},-\widetilde{z}_{1}^{*}\right) \in \widehat{N}\left(\left(x_{1}, \widetilde{z}_{1}\right)\right.$; epi $\left.F\right)$ with $\left(x_{1}, \widetilde{z}_{1}\right) \in \operatorname{gph} F$. Taking into account that $-\widetilde{z}_{1}^{*} \in N(0 ; \Theta)$ with $\left\|\widetilde{z}_{1}^{*}\right\|=1$, we arrive at the required relationship (4.28) by definition (2.8) of the Fréchet subdifferential for set-valued mappings.

Now add the index " $k$ " to indicate the sequences $\left(x_{1 k}, \widetilde{z}_{1 k}\right)$ and $\left(\widetilde{x}_{1 k}^{*}, \widetilde{z}_{1 k}^{*}\right), k \in \mathbb{N}$, defined in (4.26) and (4.28). Using estimates (4.21) and (4.25) as $k \rightarrow \infty$, we get therefore

$$
\left(x_{1 k}, \tilde{z}_{1 k}\right) \in \operatorname{gph} F \text { and } \widetilde{x}_{1 k}^{*} \in \widehat{\partial} F\left(x_{1 k}, \widetilde{z}_{1 k}\right) \text { as } k \in \mathbb{N} \text { with }\left\|\widetilde{x}_{1 k}^{*}\right\| \rightarrow 0 \text { as } k \rightarrow \infty .
$$

Employing finally the refined subdifferential Palais-Smale condition of Definition 4.2, we get from (4.32) that the sequence $\left\{x_{1 k}\right\}$ contains a convergent subsequence. Since

$$
\left\|x_{k}-x_{1 k}\right\| \leq\left\|x_{k}-\widetilde{x}_{k}\right\|+\left\|\widetilde{x}_{k}-x_{1 k}\right\| \leq k^{-1}+k^{-1} \text { for all } k \in \mathbb{N}
$$

by (4.15) and (4.19), we conclude that the sequence $\left\{x_{k}\right\}$ constructed in (4.7) and (4.8) also contains a convergence subsequence. This completes the proof of the theorem.

Next we present efficient consequences of Theorem 4.3 enduring the existence of relative minimizers and weak minimizers to set-valued mappings. 
Corollary 4.4 (existence of primary relative minimizers). Suppose in addition to the assumptions of Theorem 4.3 that $\mathrm{ri} \Theta \neq \emptyset$. Then there is a relative minimizer to the set-valued mapping $F: X \rightrightarrows Z$ under consideration.

Proof. Since ri $\Theta \subset$ iri $\Theta$, Theorem 4.3 ensures the existence of intrinsic relative minimizers to $F$ provided that ri $\Theta \neq \emptyset$. Furthermore, the latter assumption implies that (primary) relative minimizers and intrinsic relative minimizers agree for any $F$ as discussed above.

Corollary 4.5 (existence of weak Pareto minimizers). Suppose in addition to the assumptions of Theorem 4.3 that int $\Theta \neq \emptyset$. Then there is a weak Pareto minimizer to the set-valued mapping $F: X \rightrightarrows Z$ under consideration.

Proof. Theorem 4.3 guarantees the existence of an intrinsic relative minimizer to $F$ provided that $\emptyset \neq \operatorname{int} \Theta \subset \operatorname{iri} \Theta$, which is surely a weak Pareto minimizer to this mapping.

Remark 4.6 (comparison with known existence theorems). We are not familiar with any existence theorems for primary relative minimizers and intrinsic relative minimizers to setvalued mappings and their specifications in either finite-dimensional or infinite-dimensional spaces. Regarding Corollary 4.5 on the existence of weak minimizers, let us mention the following three advantages of this result in comparison with our previous one in [3, Theorem 4.3]:

- As we discussed, the refined subdifferential Palais-Smale condition from Definition 4.2 is strictly better than the basic one (4.5) introduced in [3] and used in the proof of Theorem 4.3 therein.

- We improve the domination property used in [3, Theorem 4.3] by the more relaxed limiting monotonicity condition in the new Corollary 4.5.

- The pointedness requirement on the ordering cone $\Theta$ from [3, Theorem 4.3], which is equivalent to say that the cone $\Theta$ does not contain a linear subspace, is superseded now by the essentially more general condition $\Theta \backslash(-\Theta) \neq \emptyset$, which means that $\Theta$ itself is not a linear subspace of $Z$. Note that there are various ordering relations, important for both the theory and applications, which are generated by convex and non-pointed cones; see, e.g., [18, Example 5.57] regarding lexicographical multiobjective optimization.

As mentioned above, the multiobjective problem of minimizing a general set-valued mapping $F: X \rightrightarrows Z$ implicitly contains constrains given by $x \in \operatorname{dom} F$. Furthermore, we can easily reduce problems with explicit constraints of the type

$$
\text { minimize } F(x) \text { subject to } x \in \Omega \subset X
$$

and their specifications to minimizing the restriction of $F$ to the set $\Omega$ defined by

$$
F_{\Omega}(x):= \begin{cases}F(x) & \text { if } x \in \Omega \\ \emptyset & \text { otherwise. }\end{cases}
$$

Observe that problem (4.33) can be represented in the equivalent unconstrained form

$$
\text { minimize } F_{\Omega}(x)=F(x)+\Delta(x ; \Omega)
$$

involving the summation of the original cost mapping $F$ with the indicator mapping of the set $\Omega \subset X$ defined by $\Delta(x ; \Omega):=0$ if $x \in \Omega$ and $\Delta(x ; \Omega):=\emptyset$ otherwise.

To establish the existence of optimal solutions (Pareto-type minimizers under consideration) for the constrained problem (4.33), we can apply Theorem 4.3 to the summation mapping $F_{\Omega}$ in 
(4.35) and then to proceed by using a subdifferential sum rule in the settings (4.5) and (4.6) of both Palais-Smale conditions to derive verifiable existence results in terms of the initial data $F$ and $\Omega$ of the constrained problem (4.33). From this viewpoint, the basic subdifferential Palais-Smale condition (4.5), being generally more restrictive than the refined one (4.6), has visible advantages in comparison with (4.6) due to the much more robust pointwise calculus available for the limiting constructions (2.2), (2.4), and (2.9) in comparison with their Fréchet-like counterparts; cf. [17].

Employing this calculus in the way developed in [18, Chapter 5] and [4, Section 3], we can derive the corresponding consequences of Theorem 4.3 and its Corollaries 4.4 and 4.5 to the existence theorems for multiobjective problems with geometric constraints as in (4.33) and with other types of constraints-functional, operator, and particularly of the equilibrium type considered in $[4$, Theorem 3.4] for the case of weak Pareto minimizers. Note that the latter paper in the only one, to the best of our knowledge, where the generalized differential calculus has been employed for deriving existence theorems in optimization problems (with either scalar or vector/set-valued objectives), in contrast to more conventional settings of necessary optimality conditions as in numerous publications; see, e.g., the book [18] and the references therein. This approach, which we are not going to develop here, can be well applied in the similar way for establishing verifiable existence theorems of relative Pareto minimizers to constrained multiobjective problems involving the new Theorem 4.3 under the basic subdifferential Palais-Smale condition (4.5).

In what follows we intend to explore in more detail the possibility to employ in constrained multiobjective problems the above Theorem 4.3 under the refined Palais-Smale condition (4.6) in terms of the Fréchet-type subdifferential (2.8). It has been well recognized that Fréchet-type subdifferential constructions possess generally a poor pointwise calculus, even in simple finitedimensional settings. Nevertheless, it has been recently discovered [19] that such a pointwise calculus can be developed under appropriate assumptions for some classes of extended-real valued functions. Quite recently, certain vector counterparts of these results have been derived in [1].

Given a single-valued mapping $f: X \rightarrow Z$ between Banach spaces with the ordering cone $\Theta$ of $Z$, observe that its Fréchet subdifferential (2.8) can be represented as

$$
\widehat{\partial} f(\bar{x})=\bigcup_{\substack{-z^{*} \in N(0 ; \ominus) \\\left\|z^{*}\right\|=1}} \widehat{\partial} f(\bar{x})\left(z^{*}\right), \text { where } \widehat{\partial} f(\bar{x})\left(z^{*}\right):=\widehat{D}^{*} \mathcal{E}_{f}(\bar{x}, f(\bar{x}))\left(z^{*}\right) .
$$

It follows from $[1$, Theorem 5.3] that

$$
\widehat{\partial}(f+\Delta)(\bar{x})\left(z^{*}\right) \subset \bigcap_{v \in \hat{\partial}(-f)(\bar{x})\left(z^{*}\right)}[\widehat{N}(\bar{x} ; \Omega)-v]
$$

provided that $\widehat{\partial}(-f)(\bar{x})\left(z^{*}\right) \neq \emptyset$ and that there is a neighborhood $U$ of $\bar{x}$ as well as nonnegative numbers $l$ and $m$ such that

$$
\|f(u)-f(\bar{x})\| \leq l\|u-\bar{x}\|+m\left|\left\langle z^{*}, f(u)-f(\bar{x})\right\rangle\right| \text { for all } u \in U .
$$

Note that condition (4.38) automatically holds if either $Z=\mathbb{R}$, or the mapping $f$ is upper Lipschitzian at $\ddot{x}$ that corresponds to $m=0$ in (4.38) and is surely fulfilled when $f$ is locally Lipschitzian around this points.

The next theorem ensures the existence of intrinsic relative minimizers to the constrained multiobjective problem (4.33) as well as the existence of relative Pareto and weak Pareto minimizers to (4.33) under additional assumptions. 
Theorem 4.7 (existence of relative and weak Pareto minimizers to constrained multiobjective problems). Let the mapping $f: X \rightarrow Z$ and the ordering cone $\theta \subset Z$ satisfy the general assumptions of Theorem 4.3, and let $\Omega \subset X$ be closed. Assume in addition that $\widehat{\partial}(-f)(x)\left(z^{*}\right) \neq \emptyset$ and condition (4.38) holds for any $x \in \Omega$ and $z^{*} \in-N(0 ; \Theta)$ with $\left\|z^{*}\right\|=1$ and that every sequence $\left\{x_{k}\right\} \subset \Omega$ with

$$
\left\{\begin{array}{c}
\exists x_{k}^{*} \in \bigcap_{v \in \hat{\partial}(-f)\left(x_{k}\right)\left(z_{k}^{*}\right)}\left[\widehat{N}\left(x_{k} ; \Omega\right)-v\right] \text { with }-z_{k}^{*} \in N(0 ; \Theta),\left\|z_{k}^{*}\right\|=1, k \in \mathbb{N}, \\
\text { and }\left\|x_{k}^{*}\right\| \rightarrow 0 \text { as } k \rightarrow \infty
\end{array}\right.
$$

contains a convergent subsequence. Then problem (4.33) admits an intrinsic relative minimizer provided that iri $\Theta \neq \emptyset$. Furthermore, this problem admits a primary relative Pareto minimizer if ri $\Theta \neq \emptyset$, and it admits a weak Pareto minimizer if $\operatorname{int} \Theta \neq \emptyset$.

Proof. Considering the equivalent unconstrained form (4.35) of problem (4.33), it is easy to see that the iestriction mapping $f_{\Omega}$ from (4.34) satisfies all the assumptions of Theorem 4.3 except the refined subdifferential Palais-Smale condition from Definition 4.2, which should be verified. To do it, take sequences $\left\{x_{k}\right\},\left\{x_{k}^{*}\right\}$ satisfying (4.6) for $F=f_{\Omega}$ and by (4.36) find $\left\{z_{k}^{*}\right\}$ such that

$$
x_{k}^{*} \in \widehat{\partial}[f+\Delta(\cdot ; \Omega)]\left(x_{k}\right)\left(z_{k}^{*}\right),-z_{k}^{*} \in N(0 ; \Theta),\left\|z_{k}^{*}\right\|=1 \text { with }\left\|x_{k}^{*}\right\| \rightarrow 0 \text { as } k \rightarrow \infty,
$$

which implies that $\left\{x_{k}\right\} \subset \Omega$ for all $k \in \mathbb{N}$. Employing now the subdifferential sum rule (4.37) in (4.40) under the assumptions made in the theorem, we get

$$
x_{k}^{*} \in \bigcap_{v \in \hat{\partial}(-f)\left(x_{k}\right)\left(z_{k}^{*}\right)}\left[\widehat{N}\left(x_{k} ; \Omega\right)-v\right] \text { with }\left\|x_{k}^{*}\right\| \rightarrow 0 \text { as } k \rightarrow \infty,
$$

where $-z_{k}^{*} \in N(0 ; \Theta)$ and $\left\|z_{k}^{*}\right\|=1$ for all $k \in \mathbb{N}$, i.e., the triple $\left\{x_{k}, x_{k}^{*}, z_{k}^{*}\right\}$ satisfies (4.39). Thus the sequence $\left\{x_{k}\right\} \subset \Omega$ contains a convergent subsequence, which verifies the refined Palais-Smale condition (4.6) for the restriction mapping $f_{\Omega}$ and hence ensures the existence of intrinsic relative minimizers to (4.33) provided that iri $\Theta \neq \emptyset$. The existence of primary relative Pareto minimizers and weak Pareto minimizers to (4.33) provided that $\operatorname{ri} \Theta \neq \emptyset$ and $\operatorname{int} \Theta \neq \emptyset$, respectively, is justified similarly to the proofs of Corollaries 4.4 and 4.5 .

The major assumptions of Theorem 4.7 are automatically fulfilled and/or significantly simplified when the cost mapping $f$ is Fréchet differentiable on $\Omega$.

Corollary 4.8 (existence of relative and weak Pareto minimizers to constrained multiobjective problems with Fréchet differentiable objectives). Let the mapping $f: X \rightarrow Z$ and the ordering cone $\theta \subset Z$ satisfy the general assumptions of Theorem 4.3, let $\Omega \subset X$ be closed, and let $f$ be Fréchet differentiable on $\Omega$. Assume also that every sequence $\left\{x_{k}\right\} \subset \Omega$ such that

$$
\exists x_{k}^{*} \in \nabla f\left(x_{k}\right)^{*} z_{k}^{*}+\widehat{N}\left(x_{k} ; \Omega\right) \text { with }-z_{k}^{*} \in N(0 ; \Theta),\left\|z_{k}^{*}\right\|=1, \text { and }\left\|x_{k}^{*}\right\| \rightarrow 0
$$

as $k \rightarrow \infty$ contains a convergence subsequence. Then problem (4.33) admits an intrinsic relative minimizer provided that iri $\Theta \neq \emptyset$. Furthermore, (4.33) admits a primary relative Pareto minimizer if ri $\Theta \neq \emptyset$, and it admits a weak Pareto minimizer if int $\Theta \neq \emptyset$.

Proof. It easily follows from (2.5) and (2.8) that

$$
\widehat{\partial}(-f)(x)\left(z^{*}\right)=\left\{-\nabla f(x)^{*} z^{*}\right\} \neq \emptyset
$$


for all $x$ and $z^{*}$ under consideration. Furthermore, we can directly check that the Fréchet differentiability of $f$ implies property (4.38) on $\Omega$. Thus all the assumptions of Theorem 4.7 are satisfied, and condition (4.39) reduces to (4.41) for Fréchet differentiable objectives. This justifies the conclusions of the corollary under the corresponding requirements on $\Theta$.

\section{Necessary Conditions for Pareto and Relative Pareto Minimiz- ers in Multiobjective Optimization}

In this section we establish necessary optimality conditions for all the three types of (localized) relative Pareto minimizers to multiobjective problems introduced in Definition 4.1. The results obtained in what follows provide also new necessary conditions for Pareto minimizers (efficient solutions) and weak Pareto minimizers in comparison with the previous ones from [3], where these notions have been studied for pointed ordering cones satisfying the additional "sequential normal compactness" assumption (see below) imposed in the case of Pareto minimizers in infinite dimensions. Here we derive necessary conditions in both settings of pointed and non-pointed ordering cones, where the results of the latter type occur to be of a different structure in comparison with those for the pointed case of efficient solutions.

Recall that the localized versions of all the minimizers under consideration are defined in the same way as for the global ones, where instead of all $x \in X$ (or $x \in \Omega$ for the constrained problems) we consider only those points that belong to a neighborhood of the reference solution. Thus the pair $(\bar{x}, \bar{z}) \in \operatorname{gph} F$ is a local primary/intrinsic/quasi relative minimizer to $F: X \rightrightarrows Z$ if there is a neighborhood $U$ of $\bar{x}$ such that

$$
(\bar{z}-\widetilde{\Theta}) \cap F(U)=\emptyset \text { provided that } \tilde{\Theta} \neq \emptyset,
$$

where $\widetilde{\Theta}$ stands for $\operatorname{ri} \Theta$, iri $\Theta$, and qri $\Theta$, respectively. For local weak minimizers we have (5.1) with $\widetilde{\Theta}=\operatorname{int} \Theta$. Note that the notion of local Pareto minimal/efficient solution to $F$ defined as

$$
(\bar{z}-\Theta) \cap F(U)=\{\bar{z}\}
$$

by (3.5) can be equivalently written in the form

$$
(\bar{z}-\Theta \backslash\{0\}) \cap F(U)=\emptyset
$$

similar to (5.1). This allows us to unify the proofs of necessary optimality conditions for all the local minimizers studied in the paper.

In what follows we establish in the unified way necessary conditions for all the types of relative Pareto minimizers from Definition 4.1 deriving also new results for local Pareto minimizers and weak minimizers to multiobjective problems. The results derived below are given in the same forms (different in the cases of pointed and non-pointed ordering cones) for all the types of local minimizers under consideration. Nevertheless, they are independent for the cases of quasi relative, intrinsic relative, and conventional/efficient Pareto minimizers. The necessary conditions obtained for primary relative and weak Pareto minimizers can be treated as specifications of those for quasi relative and intrinsic relative ones while, on the other hand, they are derived under much easier verifiable assumptions on the initial data due to available characterizations of the general "sequential normal compactness" requirement on $\Theta$ for the case of primary relative minimizers and due to the unconditional fulfillment of it for the case of weak efficiency. Emphasize again that the results obtained for efficient Pareto solutions do not impose any nonempty interior/relative interior assumptions on the ordering cone $\Theta$ provided that $\Theta \backslash(-\Theta) \neq \emptyset$. 
Our approach is based on reducing local Pareto/relative Pareto/weak Pareto minimizers to local extremal points of the corresponding set systems and then employing the extremal principle. As in Section 4, we first derive necessary optimality conditions for minimizing general set-valued mappings with no explicit constraints and then proceed to multiobjective problems with explicitly given constraints by using appropriate rules of subdifferential/coderivative calculus.

To obtain in this way verifiable qualification and optimality conditions in multiobjective optimization, we involve certain normal compactness properties of sets and mappings, which are automatic in finite dimensions while are unavoidably needed in infinite-dimensional spaces due to the natural lack of compactness therein. Among the major advantages of the underlying sequential normal compactness (SNC) properties presented below we emphasize extensive SNC calculus results ensuring the preservation of these properties under various operations and describing broad classes of sets and mappings in infinite dimensions for which these properties are satisfied; see the books $[17,18]$ for more details, discussions, and applications. In what follows these properties are used in the framework of Asplund spaces, and so the given definitions are specified to this setting; cf. the afore-mentioned books for appropriate modifications in general Banach spaces.

Recall that a set $\Omega \subset X$ is sequentially normally compact (SNC) at $\bar{x} \in \Omega$ if for any sequences $x_{k} \stackrel{\Omega}{\rightarrow} \bar{x}$ and $x_{k}^{*} \stackrel{w^{*}}{\rightarrow} 0$ with $x_{k}^{*} \in \widehat{N}\left(x_{k} ; \Omega\right), k \in \mathbb{N}$, we have $\left\|x_{k}^{*}\right\| \rightarrow 0$ as $k \rightarrow \infty$. A set-valued mapping $F: X \Rightarrow Z$ is $S N C$ at $(\bar{x}, \bar{z}) \in \mathrm{gph} F$ if its graph is SNC at this point. Further, we say that $F: X \rightrightarrows Z$ is partially $S N C$ (PSNC) at $(\tilde{x}, \bar{z})$ if, sequentially,

$$
\left[\left(x_{k}, z_{k}\right) \stackrel{\operatorname{gph} F}{\rightarrow}(\bar{x}, \bar{z}), x_{k}^{*} \stackrel{w^{*}}{\rightarrow} 0,\left\|z_{k}^{*}\right\| \rightarrow 0,\left(x_{k}^{*}, z_{k}^{*}\right) \in \widehat{N}\left(\left(x_{k}, z_{k}\right) ; \operatorname{gph} F\right)\right] \Longrightarrow\left\|x_{k}^{*}\right\| \rightarrow 0 \text { as } k \rightarrow \infty \text {. }
$$

The PSNC property is automatically implied by robust Lipschitzian behavior of set-valued and single-valued mappings; in particular, when $F$ is Lipschitz-like/Aubin around $(\bar{x}, \bar{z})$ with some modulus $\ell \geq 0$, i.e., there are neighborhoods $U$ of $\bar{x}$ and $V$ of $\bar{z}$ such that

$$
F(x) \cap V \subset F(u)+\ell\|x-u\| \mathbb{B} \text { for all } x, u \in U .
$$

The latter property of $F$ is fundamental in nonlinear and variational analysis; it is in fact equivalent to the two other underlying properties for the inverse mapping $F^{-1}$ known as linear openness/covering and metric regularity around $(\bar{z}, \bar{x})$.

The next theorem can be viewed as a far-going extension of the Fermat rule/stationary principle to minimizing set-valued mappings with no explicit constraints for all the types of Pareto, weak Pareto, and relative Pareto minimizers under consideration. We derive necessary conditions of two different kinds-coderivative and subdifferential, where the pointedness requirement on the ordering cone is needed only for the subdifferential case of efficient solutions.

To formulate the theorem, recall that a linear subspace of $Z$ is finite-codimensional if it is complemented in $Z$ by a finite-dimensional space.

Theorem 5.1 (generalized Fermat rules for Pareto/relative Pareto/weak Pareto minimizers to multiobjective problems with no explicit constraints). Let $F: X \rightrightarrows Z$ be a set-valued mapping between Asplund spaces such that its graph is locally closed around the reference point while the image space $Z$ is partially ordered by a closed, convex, and proper cone $\Theta \subset Z$. Then the CODERIVATIVE condition

$$
0 \in D^{*} F(\bar{x}, \bar{z})\left(z^{*}\right) \text { with some }-z^{*} \in N(0 ; \Theta) \text { and }\left\|z^{*}\right\|=1
$$

is necessary for the local optimality of $(\bar{x}, \bar{z}) \in \operatorname{gph} F$ to $F$ in each of the following senses:

- $(\bar{x}, \bar{z})$ is a local PARETo MINIMIZER/EFFICIENT SOLUTION provided that $\Theta \backslash(-\Theta) \neq \emptyset$ and that 
either $\Theta$ is SNC at the origin or $F^{-1}$ is PSNC at $(\bar{z}, \bar{x})$.

- $(\bar{x}, \bar{z})$ is a local QUASI RELATIVE MINIMIZER provided that either $\Theta$ is SNC at the origin or $F^{-1}$ is PSNC at $(\bar{z}, \bar{x})$.

- $(\bar{x}, \bar{z})$ is a local INTRINSIC RELATIVE MINIMIZER provided that either $\Theta$ is $S N C$ at the origin or $F^{-1}$ is PSNC at $(\bar{z}, \bar{x})$.

- $(\bar{x}, \bar{z})$ is a local PRIMARY RELATIVE MINIMIZER provided that either the affine closure of $\Theta$ is finite-codimensional in $Z$ or $F^{-1}$ is PSNC at $(\bar{z}, \bar{x})$.

- $(\bar{x}, \bar{z})$ is a local WEAK PAReto Minimizer.

Furthermore, we also have the SUBDIFFERENTIAL necessary optimality condition

$$
0 \in \partial F(\bar{x}, \bar{z})
$$

in each of the above cases of (efficient, quasi relative, intrinsic relative, primary relative, weak) local minimizers $(\bar{x}, \bar{z})$ provided that the epigraph vs. graph of $F$ is closed around $(\bar{x}, \bar{z})$ and that:

- PSNC assumption on $F^{-1}$ at in the assumptions above $(\bar{z}, \bar{x})$ is replaced by the PSNC assumption on the inverse mapping $\mathcal{E}_{F}^{-1}$ to the associated epigraphical multifunction (2.7) at this point;

- the ordering cone $\Theta$ is assumed to be pointed in the case of efficient solutions.

Proof. Arguing in the unified way, take any local minimizer $(\bar{x}, \bar{z}) \in \operatorname{gph} F$ to $F$ considered in theorem and reduce it to a local extremal point of some system of sets in the product space $X \times Z$. Namely, define the sets

$$
\Omega_{1}:=\operatorname{gph} F, \quad \Omega_{2}:=X \times(\bar{z}-\Theta),
$$

which are locally closed around $(\bar{x}, \bar{z})$ due to the closedness assumptions imposed on $F$ and $\Theta$. We obviously have $(\bar{x}, \bar{z}) \in \Omega_{1} \cap \Omega_{2}$. To verify the local extremality of $(\bar{x}, \bar{z})$ for $\left\{\Omega_{1}, \Omega_{2}\right\}$, let us show that there is a sequence $\left\{c_{k}\right\} \subset Z$ with $c_{k} \rightarrow 0$ as $k \rightarrow \infty$ such that

$$
\Omega_{1} \cap\left(\Omega_{2}+\left(0, c_{k}\right)\right) \cap(U \times Z)=\emptyset, \quad k \in \mathbb{N}
$$

where $U$ is a neighborhood of $\bar{x}$ from its local minimality property. This gives the required extremality relation $(2.10)$ with $a_{k}:=\left(0, c_{k}\right) \in X \times Z$.

We construct an appropriate sequence $\left\{c_{k}\right\}$ in (5.7) by putting $c_{k}:=c / k$ as $k \in \mathbb{N}$, where $0 \neq c \in Z$ is selected in the following way for each type of local minimizers considered in the theorem - this can be done by definition of the corresponding minimizer and due to the assumption $\Theta \backslash(-\Theta) \neq \emptyset$ of the (efficient) Pareto case:

- $c \in-\Theta \backslash(-\Theta)$ if $(\bar{x}, \bar{z})$ is a local Pareto minimizer;

- $c \in-q \mathrm{ri} \Theta$ if $(\bar{x}, \bar{z})$ is a local quasi relative minimizer,

- $c \in-\operatorname{iri} \Theta$ if $(\check{x}, \bar{z})$ is a local intrinsic relative minimizer,

- $c \in-\mathrm{ri} \Theta$ if $(\bar{x}, \bar{z})$ is a local primary relative minimizer,

- $c \in-\operatorname{int} \Theta$ if $(\bar{x}, \bar{z})$ is a local weak Pareto minimizer.

Arguing by contradiction, suppose that (5.7) does not hold, i.e.,

$$
\text { there is }(x, z) \in U \times Z \text { with }(x, z) \in \Omega_{1} \cap\left(\Omega_{2}+\left(0, c_{k}\right)\right) \text {. }
$$

Then, by the construction of sets (5.6), we find some $(x, z, \theta) \in X \times Z \times Z$ such that

$$
x \in U, z \in F(x)+\theta \text { with } \theta \in \Theta \text { and } z \in \bar{z}-\Theta+c_{k}, \quad k \in \mathbb{N} \text {. }
$$


This implies, by the convexity property of the ordering cone $\Theta$, that

$$
z-\theta \in F(U) \text { and } z-\theta \in \bar{z}-\theta-\Theta+c_{k} \subset \bar{z}-\Theta+c_{k}, \quad k \in \mathbb{N} .
$$

In the case of efficient/Pareto minimizers the latter gives, by their description in (5.2) and the choice of $\left\{c_{k}\right\}$ in this case, that

$$
\bar{z}-\Theta+c_{k}=\bar{z}-\Theta+(c / k) \subset(\bar{z}-\Theta-(\Theta \backslash\{-\Theta\})) \subset(\bar{z}-(\Theta \backslash\{0\})), \quad k \in \mathbb{N} .
$$

In all the cases of the relative minimizers under consideration, as well as for weak efficient solutions to $F$, we have by the choice of $\left\{c_{k}\right\}$ that

$$
\bar{z}-\Theta+c_{k}=\bar{z}-\Theta+(c / k) \subset(\bar{z}-\Theta-\widetilde{\Theta}) \subset(\bar{z}-\widetilde{\Theta}), \quad k \in N,
$$

where $\widetilde{\Theta}$ stands for either qri $\Theta$, or iri $\Theta$, or $\operatorname{ri} \Theta$, or int $\Theta$ in the corresponding cases of local minimizers. The last inclusion in (5.11) is obvious in the case of weak minimizers while follows from $[5$, Lemma 3.1$\}$ in all the cases of relative ones. Combining the relationships in (5.8)-(5.11), we have $z-\theta \in(\bar{z}-\widetilde{\Theta}) \cap F(U)$ for relative and weak minimizers and $z-\theta \in(\bar{z}-\Theta \backslash\{0\}) \cap F(U)$ for local efficient solutions to $F$. This surely contradicts (5.1) and (5.2) and thus justifies by (5.7) the local extremality of the point $(\bar{x}, \bar{z})$ for system $\left\{\Omega_{1}, \Omega_{2}\right\}$ in all the cases under consideration.

Equip now the space $X \times Z$ with the sum norm $\|(x, z)\|:=\|x\|+\|z\|$ and observe that it is Asplund as a product of Asplund spaces. Then applying the extremal principle to the set system $\left\{\Omega_{1}, \Omega_{2}\right\}$ in (5.6) and taking into account their particular structures and the maximum form of the dual norm on $X^{*} \times Z^{*}$, for any sequence $\varepsilon_{k} \downarrow 0$ as $k \rightarrow \infty$ we find $\left\{\left(x_{i k}, z_{i k}\right)\right\} \subset X \times Z$ and $\left\{\left(x_{i k}^{*}, z_{i k}^{*}\right)\right\} \subset X^{*} \times Z^{*}$ as $i=1,2$ satisfying for all $k \in \mathbb{N}$ the following relationships:

$$
\begin{gathered}
\left(x_{1 k}, z_{1 k}\right) \in \operatorname{gph} F, \quad\left(x_{2 k}, z_{2 k}\right) \in X \times(\bar{z}-\Theta), \quad\left\|\left(x_{i k}, z_{i k}\right)-(\bar{x}, \vec{z})\right\| \leq \varepsilon_{k}, \\
\left(x_{1 k}^{*},-z_{1 k}^{*}\right) \in \widehat{N}\left(\left(x_{1 k}, z_{1 k}\right) ; \operatorname{gph} F\right), \quad 0 \equiv x_{2 k}^{*} \in \widehat{N}\left(x_{2 k} ; X\right), \quad z_{2 k}^{*} \in \widehat{N}\left(\bar{z}-z_{2 k} ; \Theta\right), \\
\max \left\{\left\|x_{1 k}^{*}\right\|,\left\|z_{1 k}^{*}+z_{2 k}^{*}\right\|\right\} \leq \varepsilon_{k}, \quad \text { and } 1-\varepsilon_{k} \leq \max \left\{\left\|x_{1 k}^{*}\right\|,\left\|z_{1 k}^{*}\right\|\right\}+\left\|z_{2 k}^{*}\right\| \leq 1+\varepsilon_{k} .
\end{gathered}
$$

By the second relationship in (5.14,) the sequences $\left\{\left(x_{i k}^{*}, z_{i k}^{*}\right)\right\}$ are bounded in $X^{*} \times Z^{*}$ for $i=1,2$, and hence-by the Asplund property of $X \times Z$-they contain weak $k^{*}$ converging subsequences; see, e.g., $[14,17]$. Using the first relationship in $(5.14)$, we get without loss of generality that

$$
\left\|x_{1 k}^{*}\right\| \rightarrow 0, \quad z_{1 k}^{*} \stackrel{w^{*}}{\rightarrow} z^{*}, \text { and } z_{2 k}^{*} \stackrel{w^{*}}{\rightarrow}-z^{*} \text { as } k \rightarrow \infty,
$$

where the weak* limit $z^{*} \in Z^{*}$ satisfies the inclusions

$$
\left(0,-z^{*}\right) \in N((\bar{x}, \tilde{z}) ; \operatorname{gph} F) \text { and }-z^{*} \in N(0 ; \Theta)
$$

obtained by passing to the limit in relationships (5.12) and (5.13) as $k \rightarrow \infty$ due to construction (2.2) of the basic normal cone via the sequential outer limit (1.9) of Fréchet normals.

Next we show that $z^{*} \neq 0$ in (5.16) if either $\Theta$ is SNC at the origin or $F^{-1}$ is PSNC at $(\bar{z}, \bar{x})$ for all the types of the local minimizers under consideration. Assume by the contrary that $z^{*}=0$ having then from (5.15) that

$$
z_{1 k}^{*} \stackrel{w^{*}}{\rightarrow} 0 \text { and } z_{2 k}^{*} \stackrel{w^{*}}{\rightarrow} 0 \text { as } k \rightarrow \infty
$$


If $\Theta$ is SNC at the origin, then the second expression in (5.17) immediately yields that $\left\|z_{2 k}^{*}\right\| \rightarrow 0$ and therefore $\left\|z_{1 k}^{*}\right\| \rightarrow 0$ as $k \rightarrow \infty$ by the first relationship in (5.14). Combining the latter with (5.15), we thus contradict the nontriviality/second expression in (5.14). Suppose now that $F^{-1}$ is PSNC at $(\bar{z}, \bar{x})$. Using the first inclusion in (5.13) and the convergence $\left\|x_{1 k}^{*}\right\| \rightarrow 0$ in (5.15), we conclude from the imposed PSNC property that $\left\|z_{1 k}^{*}\right\| \rightarrow 0$ as $k \rightarrow \infty$. This gives $\left\|z_{2 k}^{*}\right\| \rightarrow 0$ as $k \rightarrow \infty$ and also contradicts the second expression in (5.14). Therefore $z^{*} \neq 0$ in (5.16), which yields the coderivative condition (5.4) by normalization and by definition of the coderivative (2.4). Thus we arrive at the conclusions of the theorem regarding the coderivative necessary condition for the cases of Pareto minimizers, quasi relative minimizers, and intrinsic relative minimizers.

The case of primary relative minimizers requirers that ri $\Theta \neq \emptyset$. The latter allows us to fully characterize the $S N C$ property of $\Theta$. Indeed, Theorem 1.21 from [17] tells us that the SNC property of a convex set with nonempty relative interior in a Banach space is equivalent to the finite codimension of its affine closure; cf. also [8, Theorem 2.5] for the same characterization, with a completely different proof, of the generally more restrictive compactly epi-Lipschitzian property of closed and convex sets with nonempty relative interiors, that happens to be equivalent to the SNC property for such sets. This justifies the coderivative necessary condition (5.4) of the theorem in the case of primary relative minimizers.

If $(\bar{x}, \bar{z})$ is a weak Pareto minimizer to $F$, then int $\Theta \neq \emptyset$. In this case the convex ordering cone $\Theta$ is automatically $S N C$. Indeed, it is well known (see, e.g., [17, Proposition 1.25]) that the nonempty interior property of a convex set is equivalent to its epi-Lipschitzian property, which implies the SNC one by [17, Theorem 1.26]. Thus the coderivative result (5.4) unconditionally holds for weak Pareto minimizers to general set-valued mappings.

To complete the proof of the theorem, it remains to justify the subdifferential necessary condition (5.5) for all the local minimizers under consideration. Given $F: X \rightrightarrows Z$ and its epigraphical multifunction $\mathcal{E}_{F}: X \rightrightarrows Z$ from (2.6), define the auxiliary set-valued optimization problem:

$$
\text { minimize } \mathcal{E}_{F}(x)=F(x)+\Theta, \quad x \in X .
$$

It is clear that every local optimal solution to (5.18) in each of the above-mentioned sense is a local optimal solution in the corresponding sense to the mapping $F$. For our purposes we need and prove the opposite implication ensuring that a local minimizer to $F$ is a local minimizer in the same sense to the epigraphical multiobjective problem (5.18). For the case of Pareto/efficient minimizers this follows from [15, Lemma 3.14] under the assumption that the ordering cone $\Theta$ is pointed. We show below that the same holds for all the other local minimizers under consideration with no pointedness assumption. The latter follows from the fact that the underlying minimization relationship (5.1) for $F$ yields the one

$$
(\bar{z}-\widetilde{\Theta}) \cap(F(U)+\Theta)=\emptyset
$$

for (5.18), where $\widetilde{\Theta}$ stands, respectively, for each of qri $\Theta$, $\operatorname{iri} \Theta$, ri $\Theta$, and int $\Theta$. Indeed, assuming the negation of (5.19), we get $z \in(\bar{z}-\widetilde{\Theta}) \cap(F(U)+\Theta)$ and thus find

$$
u \in U, \quad v \in F(u) \text {, and } \theta \in \Theta \text { such that } z=v+\theta \subset \bar{z}-\widetilde{\Theta} \text {. }
$$

This gives the relationships

$$
v=z-\theta \in \bar{z}-\theta-\widetilde{\Theta} \subset \bar{z}-\Theta-\widetilde{\Theta} \in \bar{z}-\widetilde{\Theta}
$$

for all the cases of $\widetilde{\Theta}$ under consideration, where the latter inclusion is trivial for $\widetilde{\Theta}=\operatorname{int} \Theta$ while follows from the afore-mentioned result in [5, Lemma 3.1] for the cases of (primary, intrinsic, and 
quasi) relative minimizers: $\widetilde{\Theta}=\operatorname{ri} \Theta, \widetilde{\Theta}=\operatorname{iri} \Theta$, and $\widetilde{\Theta}=\operatorname{qri} \Theta$. Hence we get $v \in(\bar{z}-\widetilde{\Theta}) \cap F(U)$, which contradicts (5.1) and thus justifies (5.19) for the weak and relative minimizers.

We conclude therefore that any local minimizer $(\bar{x}, \bar{z})$ to $F$ considered in the theorem is also a local minimizer in the same case to the epigraphical problem (5.18) under the assumptions made. Applying finally to problem (5.18) the necessary optimality conditions justified in the first/coderivative part of the theorem and taking into account definition (2.9) of the basic subdifferential for $F$, we arrive at the subdifferential optimality condition (5.5) for all the local minimizers under consideration. This completes the proof of the theorem.

As we have already discussed in Section 4 , the multiobjective problem of minimizing a general set-valued mapping considered in Theorem 5.1 implicitly incorporates various constraints via the actual restriction $x \in \operatorname{dom} F$ of the set of feasible solutions. For example, to study in this vein the constrained set-valued optimization problem

$$
\text { minimize } F(x) \text { subject to } x \in \Omega
$$

with explicit geometric constraints given by an arbitrary nonempty set $\Omega \subset X$, we reduce it to the (formally) unconstrained problem of minimizing the restriction mapping $F_{\Omega}(x)=F(x)+\Delta(x ; \Omega)$ as in (4.34) and (4.35). To derive in this way verifiable necessary optimality conditions for the constrained problem $(5.20)$, we need to apply calculus rules to the summation mapping $F+\Delta(\cdot ; \Omega)$ that allow us to express the generalized differential and SNC assumptions and conclusions of Theorem 5.1 in terms of the initial data of problem (5.20). Full calculi for both generalized differential constructions and SNC properties under consideration are available in Asplund spaces; see [17, Chapter 3], where calculus results of both types are derived from the extremal principle.

To obtain the strongest results in this direction for the constrained problem (5.20), we need to involve two more coderivative/subdifferential constructions for set-valued mappings in addition to those presented in Section 2. Given $F: X \rightrightarrows Z$ and $(\bar{x}, \bar{z}) \in \operatorname{gph} F$, recall that the mixed coderivative of $F$ at $(\bar{x}, \bar{z})$ is defined by

$$
\begin{aligned}
D_{M}^{*} F(\bar{x}, \bar{z})\left(z^{*}\right):=\left\{x^{*} \in X^{*} \mid\right. & \exists \text { sequences }\left(x_{k}, z_{k}\right) \stackrel{\operatorname{gph} F}{\rightarrow}(\bar{x}, \bar{z}), x_{k}^{*} \stackrel{w^{*}}{\rightarrow} x^{*}, z_{k}^{*} \rightarrow z^{*} \\
& \text { with } \left.\left(x_{k}^{*},-z_{k}^{*}\right) \in \widehat{N}\left(\left(x_{k}, z_{k}\right) ; \operatorname{gph} F\right), \quad k \in \mathbb{N}\right\} .
\end{aligned}
$$

Taking into account the basic normal cone definition (2.2), we easily observe that the only difference between the mixed coderivative (5.21) and the basic/normal coderivative (2.4) is that the strong convergence of $\left\|z_{k}^{*}-z^{*}\right\| \rightarrow 0$ is employed in (5.21) instead of the weak sequential convergence $z_{k}^{*} \stackrel{w^{*}}{\rightarrow} z^{*}$ in (2.4), while the weak* convergence $x_{k}^{*} \stackrel{w^{*}}{\rightarrow} x^{*}$ is used in both cases. This immediately implies the inclusion

$$
D_{M}^{*} F(\bar{x}, \bar{z})\left(z^{*}\right) \subset D^{*} F(\bar{x}, \bar{z})\left(z^{*}\right) \text { for all } z^{*} \in Z^{*},
$$

where the equality holds when $\operatorname{dim} Z<\infty$. By (2.5), the equality holds in (5.22) as well when the mapping in question is strictly differentiable at $\bar{x}$; it also holds in some other cases listed in [17, Proposition 4.9], while in general the inclusion is strict in (5.22) even for single-valued and Lipschitz continuous mappings from $\mathbb{R}$ to a Hilbert space as in [17, Example 1.35]. A significant advantage of the mixed coderivative is that

$$
D_{M}^{*} F(\bar{x}, \bar{z})(0)=\{0\}
$$

when $F$ exhibits robust Lipschitzian behavior expressed in the general way (5.3) as the Lipschitzlike/Aubin property of $F$ around $(\bar{x}, \bar{z})$. 
In what follows we also need the construction

$$
\partial^{\infty} F(\bar{x}, \bar{z}):=D_{M}^{*} \mathcal{E}_{F}(\bar{x}, \bar{z})(0)
$$

of the singular subdifferential of mappings with values in partially ordered spaces defined via the mixed coderivative (5.21) of the corresponding epigraphical multifunction $\mathcal{E}_{F}$ from (2.6); cf. $[3,17]$ for related discussions and properties.

The next theorem presents necessary optimality conditions for local minimizers of all the types under consideration in the multiobjective problem (5.20) with general geometric constraints.

Theorem 5.2 (necessary conditions for local minimizers to constrained multiobjective problems). Let $F: X \rightrightarrows Z$ be a set-valued mapping between Asplund spaces with the image space $Z$ partially ordered by a closed, convex, and proper cone $\Theta \subset Z$. Suppose that the constraint set $\Omega \subset X$ is locally closed around the reference local minimizer $(\bar{x}, \bar{z})$ to $(5.20)$. The following assertions hold:

(i) Assume that the graph of $F$ is locally closed around $(\vec{x}, \bar{z})$, that the qualification condition

$$
D_{M}^{*} F(\bar{x}, \bar{z})(0) \cap(-N(\bar{x} ; \Omega))=\{0\}
$$

is satisfied, and that either $F$ is PSNC at $(\bar{x}, \bar{z})$ or $\Omega$ is SNC at $\bar{x}$; both the qualification condition (5.25) and the PSNC property of $F$ are automatically satisfied if $F$ is Lipschitz-like around $(\bar{x}, \bar{z})$. Then there exists $-z^{*} \in N(0 ; \Theta)$ with $\left\|z^{*}\right\|=1$ such that

$$
0 \in D^{*} F(\bar{x}, \bar{z})\left(z^{*}\right)+N(\bar{x} ; \Omega)
$$

in each of the following cases of local minimizers to (5.20);

- $(\bar{x}, \bar{z})$ is a local PARETO MINIMIZER/EFFICIENT SOLUTION provided that $\Theta \backslash(-\Theta) \neq \emptyset$ and that either $\Theta$ is SNC at the origin or $F_{\Omega}^{-1}$ is PSNC at $(\bar{z}, \bar{x})$.

- $(\bar{x}, \bar{z})$ is a local QUASI RELATIVE MINIMIZER provided that either $\Theta$ is SNC at the origin or $F_{\Omega}^{-1}$ is PSNC at $(\bar{z}, \bar{x})$.

- $(\bar{x}, \bar{z})$ is a local INTRINSIC RELATIVE MINIMIZER provided that either $\Theta$ is SNC at the origin or $F_{\Omega}^{-1}$ is PSNC at $(\bar{z}, \bar{x})$.

- $(\bar{x}, \bar{z})$ is a local PRIMARY RELATIVE MINIMIZER provided that either the affine closure of $\Theta$ is finite-codimensional in $Z$ or $F_{\Omega}^{-1}$ is PSNC at $(\bar{z}, \bar{x})$.

- $(\bar{x}, \bar{z})$ is a local WEAK PARETO Minimizer.

(ii) Assume that $F$ is epiclosed around $(\bar{x}, \bar{z})$, that the qualification condition

$$
\partial^{\infty} F(\bar{x}, \bar{z}) \cap(-N(\bar{x} ; \Omega))=\{0\}
$$

is satisfied, and that-just in the case of efficient solutions-the ordering cone $\Theta$ is pointed. Then we have the subdifferential necessary optimality condition

$$
0 \in \partial F(\bar{x}, \bar{z})+N(0 ; \Omega)
$$

for all the local minimizers considered in assertion (i) provided that the assumptions on $F$ in (i) are replaced by the corresponding assumptions on its epigraphical multifunction $\mathcal{E}_{F}$. 
Proof. To justify assertion (i), we reduce the constrained problem (5.20) to the equivalent multiobjective problem (4.35) with no explicit constraints by using the restriction mapping $F_{\Omega}$ from (4.34). Applying now Theorem 5.1 to the mapping $F_{\Omega}$ for all the types of local minimizers under consideration, we find $z^{*} \in-N(0 ; \Theta)$ with $\left\|z^{*}\right\|=1$ such that

$$
0 \in D^{*} F_{\Omega}(\vec{x}, \bar{z})\left(z^{*}\right)=D^{*}(F+\Delta(\cdot ; \Omega))(\bar{x}, \bar{z})\left(z^{*}\right)
$$

Then we use the coderivative sum rule in (5.28) from [17, Proposition 3.12], which gives

$$
D^{*}(F+\Delta(\cdot ; \Omega))(\bar{x}, \bar{z})\left(z^{*}\right) \subset D^{*} F(\bar{x}, \bar{z})\left(z^{*}\right)+N(\bar{x} ; \Omega)
$$

under the qualification condition (5.25) and the SNC/PSNC requirements on $(F, \Omega)$ imposed in the theorem. Substituting (5.29) into (5.28) and taking into account the assumptions of Theorem 5.1 made for each type of local minimizers under consideration, we arrive at the corresponding conclusions of assertion (i).

To prove assertion (ii), observe that the assumptions therein ensure that every local minimizer $(\bar{x}, \bar{z})$ to $(5.20)$ is a local minimizer of the same type to the epigraphical problem

$$
\text { minimize } \mathcal{E}_{F}(x) \text { subject to } x \in \Omega \text {; }
$$

see the proof of Theorem 5.1. Applying now the results of assertion (i) to problem (5.30) and using the above definitions of the basic and singular subdifferentials to $F$ at $(\bar{x}, \bar{z})$ from $(2.9)$ and (5.24), respectively, we arrive at the subdifferential condition (5.27) under the assumptions made in (ii). This completes the proof of the theorem.

Observe that while the qualification and optimality conditions obtained in Theorem 5.2 are expressed directly via robust pointbased constructions for the initial data $(F, \Theta, \Omega)$, the assumptions imposed in the cases of Pareto and relative Pareto minimizers involve the PSNC property of the mapping $F_{\Omega}^{-1}$ and its epigraphical counterpart that are not automatic when $\operatorname{dim} Z=\infty$. The next result presents necessary optimality conditions for (5.20), where the PSNC requirements are specified given in terms of $F$ and $\Omega$ but not via their combination $F_{\Omega}$. This is a consequence of the SNC calculus developed in [17]. For simplicity, we formulate a corollary of Theorem 5.2 only for the case of assertion (i) therein. Note that the qualification condition imposed in this corollary is more restrictive (in the case of $\operatorname{dim} Z<\infty$ ) than the one (5.25) in the theorem, but it allows us to establish the required result entirely in terms of the initial data.

Corollary 5.3 (optimality conditions for constrained multiobjective problems under specified assumptions). Let the qualification condition (5.25) in Theorem 5.2(i) be replaced by

$$
D^{*} F(\bar{x}, \bar{z})(0) \cap(-N(\bar{x} ; \Omega))=\{0\},
$$

and let the PSNC assumption on $F_{\Omega}^{-1}$ be replaced by

- either $F^{-1}$ is PSNC at $(\bar{z}, \bar{x})$ and $\Omega$ is SNC at $\bar{x}$;

- or $F$ is $S N C$ at $(\bar{x}, \bar{z})$.

Then condition (5.26) with some $z^{*} \in-N(0 ; \Theta)$ and $\left\|z^{*}\right\|=1$ is necessary for optimality in the all cases of local minimizers under consideration.

Proof. To justify this statement, we need to check that the qualification condition (5.31) and either one of the alternative assumptions made in the corollary imply that $F_{\Omega}^{-1}$ is PSNC at $(\bar{z}, \bar{x})$. To proceed, observe that the PSNC property of the mapping $F_{\Omega}^{-1}$ at $(\bar{z}, \bar{x})$ is equivalent to the PSNC property at this point of the set $\mathrm{gph} F_{\Omega}$ in the product space $X \times Z$ with respect to $Z$ in 
the sense of [17, Definition 3.3]. Since gph $F_{\Omega}^{-1}=\operatorname{gph} F \cap(\Omega \times Z)$, we apply the intersection rule for the PSNC property from [17, Corollary 3.80] to the sets $\Omega_{1}:=\operatorname{gph} F$ and $\Omega_{2}:=\Omega \times Z$, which gives us the required result due to the specific structures of $\Omega_{1}$ and $\Omega_{2}$.

Remark 5.4 (comparison with related necessary optimality conditions in multiobjective optimization). Being new for the cases of relative minimizers under consideration, the necessary optimality conditions obtained in this section give significant improvements over recent results concerning Pareto/efficient minimizers and weak minimizers to multiobjective problems. Note that necessary optimality conditions for Pareto minimizers close to assertion (i) of Theorem 5.2 are obtained in [23, Theorem 4.1] under a stronger qualification condition in comparison with (5.25) assuming also that the ordering cone $\Theta$ satisfies a certain "dual compactness" requirement, which surely implies the SNC property of $\Theta$ imposed in Theorem 5.2, with no alternative assumptions on $F$ in [23] when $\Theta$ fails to be dually compact. In [4, Theorem 4.1 and Remark 4.2] (cf. also [3] for single-valued objectives), we derive necessary optimality conditions of the subdifferential type (5.27) as in assertion (ii) of Theorem 5.2 for Pareto and weak Pareto minimizers assuming that the ordering cone $\Theta$ is pointed and SNC at the origin, with no alternative requirements on $F$ if it is not the case.

It is worth mentioning that the notions of local minimizers under consideration can be treated as particular cases of local extremal points of sets (which has actually been shown in the proof of Theorem 5.1), and thus the results of [18, Section 5.3] concerning the generalized order optimality as well as the one defined by closed preference relations are applicable, under appropriate assumptions, to deriving necessary optimality conditions for the local minimizers considered in this paper to problems with single objectives. However, the results that could be derived in this way from [18, Theorem 5.59 and Theorem 5.73] require more restrictive assumptions in comparison with those in Theorem 5.1 and Theorem 5.2 obtained above.

Remark 5.5 (necessary optimality conditions for multiobjective problems with structural constraints). We pay the main attention in this section to problems with general geometric constraints just for brevity and simplicity. As it has been demonstrated in $[2,3,4,18]$, results obtained for single-objective and multiobjective problems with geometric constraints can be directly employed in deriving necessary optimality conditions for various classes of problems with structural constraints of functional, operator, equilibrium, and other types. This is based on welldeveloped calculi of the generalized differential constructions and SNC properties involved in the necessary optimality and qualification conditions of the results established in this paper and in the afore-mentioned publications. We also refer the reader to the papers [22, 23], where necessary optimality conditions for multiobjective problems with various constraints are derived by using somewhat different approaches based nevertheless on the ideas close to the extremal principle.

Remark 5.6 (suboptimality conditions for multiobjective problems). This section is addressed to deriving necessary optimality conditions for different kinds of local minimizers to multiobjective problems. Having in hand the subdifferential variational principle established in Theorem 3.6 and following the lines developed in [18, Subsection 5.1.4] and in $[3,20]$ in singleobjective and multiobjective frameworks, we can also derive suboptimality (or $\varepsilon$-optimality) conditions for approximately optimal solutions to the multiobjective problems under consideration that are naturally given in "fuzzy" forms while do not require the existence of minimizers.

\section{References}

[1] T. Q. Bao; On Fréchet subdifferential sum rule and its applications, preprint (2007). 
[2] T. Q. Bao, P. Gupta and B. S. Mordukhovich, Necessary conditions in multiobjective optimization with equilibrium constraints, J. Optim. Theory Appl. 135 (2007), 179-203.

[3] T. Q. Bao and B. S. Mordukhovich, Variational principles for set-valued mappings with applications to multiobjective optimization, Control and Cybernetics 36 (2007), 531-562.

[4] T. Q. Bao and B. S. Mordukhovich, Existence of minimizers and necessary conditions in set-valued optimization with equilibrium constraints, Appl. Math. 26 (2007), 453-472.

[5] J. M. Borwein and R. Goebel, Notions of relative interior in Banach spaces, J. Math. Sci. 115 (2003), $2542-2553$.

[6] J. M. Borwein and A. S. Lewis, Partially finite convex programming, Part I: Quasi relative inteiors and duality theory, Math. Prog. 57 (1992), 15-48.

[7] J. M. Borwein and A. S. Lewis, Partially finite convex programming, Part II: Explicit lattice models, Math. Prog. 57 (1992), 49-83.

[8] J. M. Borwein, Y. Lucet and B. S. Mordukhovich, Compactly epi-Lipschitzian convex sets and functions in normed spaces, J. Convex Anal. 7 (2000), 375-393.

[9] J. M. Borwein and Q. J. Zhu, Techniques of Variational Analysis, CMS Books in Mathematics 20, Springer, New York, 2005.

[10] M. Durea, J. Dutta and C. Tammer, Lagrange multipliers for $\varepsilon$-Pareto solutions in vector optimization with non-solid cones in Banach spaces, preprint (2007).

[11] I. Ekeland, On the variational principle, J. Math. Anal. Appl. 47 (1974), 324-353.

[12] A. Göpfert, H. Riahi, C. Tammer and C. Zalinescu, Variational Methods in Partially Ordered Spaces, CMS Books in Mathematics 17, Springer, New York.

[13] V. V. Gorokhovik, Convex and Nonsmooth Problems of Vector Optimization, Navuka i Tekhnika, Minsk, 1990.

[14] R. B. Holmes, Geometric Functional Analysis and Its Applications, Graduate Texts in Mathematics 24, Springer, New York, 1975.

[15] J. Jahn, Vector Optimization: Theory, Applications and Extensions, Series in Operations Research and Decision Theory, Springer, New York, 2004.

[16] D. T. Luc, Theory of Vector Optimization, Lecture Notes Econ. Math. Syst. 319, Springer, Berlin, 1989.

[17] B. S. Mordukhovich, Variational Analysis and Generalized Differentiation, I: Basic Theory, Grundlehren Series (Fundamental Principles of Mathematical Sciences) 330, Springer, Berlin, 2006.

[18] B. S. Mordukhovich, Variational Analysis and Generalized Differentiation, II: Applications, Grundlehren Series (Fundamental Principles of Mathematical Sciences) 331, Springer, Berlin, 2006.

[19] B. S. Mordukhovich, N. M. Nam and N. D. Yen, Fréchet subdifferential calculus and optimality necessary conditions in nondifferentiable programming, Optimization 55 (2006), 685-708.

[20] B. S. Mordukhovich and B. Wang, Necessary optimality and suboptimality conditions in nondifferentiable programming via variational principles, SIAM J. Control Optim. 41 (2002), 623-640.

[21] R. T. Rockafellar and R. J-B. Wets, Variational Analysis, Grundlehren Series (Fundamental Principles of Mathematical Sciences) 317, Springer, Berlin, 1998.

[22] J. J. Ye and Q. J. Zhu, Multiobjective optimization problems with variational inequality constraints, Math. Prog. 96 (2003), 139-160.

[23] X. Y. Zheng and K. F. Ng, The Lagrange multiplier rule for multifunctions in Banach spaces, SIAM J. Optim. 17 (2006), 1154-1175. 